Acta Crystallographica Section A

Foundations of Crystallography

ISSN 0108-7673

Received 2 September 2005

Accepted 9 November 2005

(C) 2006 International Union of Crystallography Printed in Great Britain - all rights reserved

\section{Groupoid of orientational variants}

\author{
Cyril Cayron
}

CEA-Grenoble DRT/LITEN, 17 rue des Martyrs, 38054 Grenoble, France. Correspondence e-mail:
cyril.cayron@cea.fr

Daughter crystals in orientation relationship with a parent crystal are called variants. They can be created by a structural phase transition (Landau or reconstructive), by twinning or by precipitation. Internal and external classes of transformations defined from the point groups of the parent and daughter phases and from a transformation matrix allow the orientations of the distinct variants to be determined. These are algebraically identified with left cosets and their number is given by the Lagrange formula. A simple equation links the numbers of variants of the direct and inverse transitions. The equivalence classes on the transformations between variants are isomorphic to the double cosets (operators) and their number is given by the Burnside formula. The orientational variants and the operators constitute a groupoid whose composition table acts as a crystallographic signature of the transition. A general method that determines if two daughter variants can be inherited from more than one parent crystal is also described. A computer program has been written to calculate all these properties for any structural transition; some results are given for Burgers transitions and for martensitic transitions in steels. The complexity, irreversibility and entropy of fractal systems constituted by orientational variants generated by thermal cycling are briefly discussed.

\section{Introduction}

Phase-transition materials are constituted by grains of the same phase (for example $\beta$ ) that, when some thermodynamical conditions are changed, are transformed into many crystals of the same phase (for example $\alpha$ ) with an orientation relationship (OR). These daughter crystals are called variants. The physical and mechanical properties of phase-transition materials highly depend on the microstructure formed by these variants. For example: (i) the martensite laths obtained by quenching or rapid cooling increase significantly the yield strength of steels; (ii) the twins that appear during deformation or recrystallization allow a stress relaxation in copper; or (iii) the nanoprecipitates that nucleate during ageing treatments give a structural hardening to aluminium alloys. These cases and many more (ferroelectric, ferroelastic and magnetic domains) have been treated in different fields of materials science with different approaches.

The aim of the present study is to show that a unique crystallographic description of these different situations can be given by using a unifying algebraic structure called a groupoid. Groupoids were first introduced to determine the quadratic forms of a given norm (Brandt, 1926). As for groups, they have a direct analogy in geometry. They can be used in many scientific domains because, as clearly pointed out by Stewart (2004), they are 'more flexible and often more appropriate than the better-known groups'. They are actually the ideal tool for describing symmetries that apply only to parts of systems containing repeated units, such as, in our case, systems constituted by variants.

Since this study has been motivated by the understanding and the exploitation of some experimental diffraction results by electron back-scattered diffraction (EBSD) and transmission electron microscopy (TEM), only the orientations of the crystals are treated (and not their positions nor the atomic structures of the interfaces). From now on, the word 'symmetry' will refer only to orientational symmetries (and the associated point group) and the word 'variants' will refer to orientational variants. Firstly, we give a brief overview of the different crystallographic studies about variants. These studies use similar algebraic tools but most of them do not introduce explicitly the orientation relationship between the parent and the daughter crystals, and the variants are not mathematically identified. Secondly, we define the orientational variants by introducing the notions of internal and external equivalence classes on transformation matrices and by using some general algebraic tools of group theory. Direct and inverse transitions will be treated. Operators will be introduced as classes of transformations between variants. Finally, we show that the set of variants and the set of transformations that link them do not form a group but a groupoid. A method that gives the possible parent crystals from the knowledge of some daughter crystals is also described. A simple geometrical case (Fig. 1) will be used as an example during the study. However, for complex reconstructive transitions, as the geometrical considerations are limited (they 
require a great capacity for visualizing complicated threedimensional objects), a computer program has been written to calculate all the variants, the transformations between them, the different possibilities of parent crystals and the composition table of the groupoid. Its application to Burgers transitions and martensitic transitions in steels will be presented. We will briefly explain on these examples how to use the composition table of groupoids to reconstruct parent crystals from EBSD data [for more details on this application, see Cayron et al. (2006)]. The problem of the generation of highorder variants during thermal cycling and the related fundamental problems will be discussed in the last section.

We have decided to sum up the algebraic notions used in this paper in Appendices $A$ and $B$. The reader is invited to go over them to at least be familiar with the mathematical notations. Some are described by Janovec et al. (2003). The reader can also refer to Milne (2003) or to Kargapolov \& Merzliakov (1985) for an introduction to group theory.

\section{An overview of crystallographic studies of variants}

\subsection{Landau transitions}

Landau has studied the thermodynamic of phase transitions when a group-subgroup relation exists between the parent and daughter symmetries (Landau, 1937a,b; Tolédano \& Tolédano, 1987). The order parameter of the transition depends on the nature of the transition (it is an atomic position or a degree of occupancy etc.) and the theory relies on the assumption that the form of the free energy should continue to respect the symmetries of the higher symmetric phase (the phase stable at high temperature) even at temperatures lower than the transition temperature. Although restrictive, this approach was fruitful: it has permitted us to show that crystallography has a direct effect on the thermodynamics of a phase transition. In this special case of group-subgroup rela-

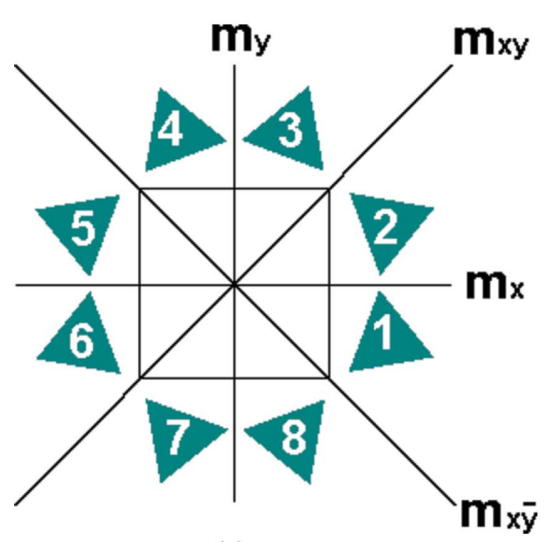

(a)

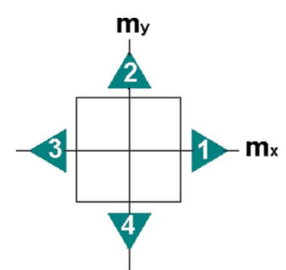

(b)

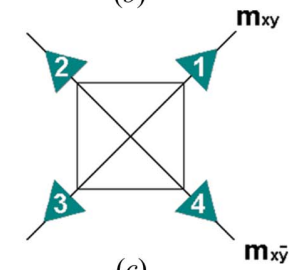

(c)
Figure 1

Two-dimensional example of variants (equilateral triangles) in orientation relationship with a parent crystal (square). The 'general' case is represented in $(a)$, there are 8 variants; the algebraic structure of the variants is a group. Two 'special' cases are represented in $(b)$ and $(c)$; the number of variants is then 4 and the algebraic structure is no longer a group but a groupoid. tionships between the parent and daughter crystals, geometrical objects called orientational domains or variants have been introduced (but not explicitly mathematically defined) from the algebraic decomposition of the symmetry group of the parent phase $\mathbf{G}$ into left cosets of the point group of one of the daughter crystals (denoted $\mathbf{H}$ or $\mathbf{F}$, depending on the authors). The algebraic developments were done for ferroelectric domains (Janovec, 1972, 1976) and for variants generated by Landau order-disorder transitions (Van Tendeloo \& Amelinckx, 1974).

Janovec introduced the term 'domain states' to refer to any physical property attached to the daughter phase crystals such as the polarization of ferroelectric domains. The first domain state is denoted $\mathbf{S}_{1}$. All the symmetry elements of $\mathbf{G}$ that leave $\mathbf{S}_{1}$ invariant form a group called the stabilizer of $\mathbf{S}_{1}$, denoted $\mathbf{F}_{1}$ (with $\mathbf{F}$ for ferroic). It was shown that the number of distinct domain states is given by the decomposition of $\mathbf{G}$ into left cosets of $\mathbf{F}_{1}$ :

$$
\mathbf{G}=g_{1} \mathbf{F}_{1} \cup g_{2} \mathbf{F}_{1} \cup \ldots \cup g_{N} \mathbf{F}_{1} .
$$

A deep algebraic study of orientational and translational variants is also given in Dirl et al. (1997) by considering the space groups of the two phases.

The algebraic base used by Van Tendeloo is similar to that of Janovec. A variant is an 'object' $\mathbf{V}_{i}$ invariant by a subgroup of $\mathbf{G}$ denoted $\mathbf{H}_{i}$, i.e. $\mathbf{H}_{i} \mathbf{V}_{i}=\mathbf{V}_{i}$. The other variants $\mathbf{V}_{j}$ are defined from $\mathbf{V}_{i}$ by an element $g$ of $\mathbf{G}$ such that $g \mathbf{V}_{i}=\mathbf{V}_{j}$ and $g \notin \mathbf{H}_{i}$. The point or space group of the parent phase is then decomposed as left (or right) cosets of $\mathbf{H}_{i}$ such as in equation (1). Van Tendeloo uses the term 'variant' to refer to a crystal oriented with reference to the parent crystal but no mathematical definition was given. Since the orientation can be considered as a crystal property, Janovec's description includes Van Tendeloo's. In both cases, the number of variants results from the Lagrange theorem: $N=|\mathbf{G}| /|\mathbf{H}|$, where $|\mathbf{G}|$ and $|\mathbf{H}|$ are the order of the groups.

\subsection{Reconstructive transitions}

In most minerals and alloys ( $\mathrm{Fe}, \mathrm{Ti}, \mathrm{Zr}$ etc.), phase transitions do not respect a group-subgroup relationship. A new thermodynamic theory of phase transition developed by Tolédano \& Dmitriev (1996) resolves this problem by generalizing the Landau approach. However, the general crystallographic and algebraic developments of these transitions are not as advanced as those presented before for the Landau transitions. The study of 'non-disruptive' transitions ${ }^{\mathbf{1}}$ introduced by Guymont (1981) tries to reduce the gap: the special case of phase transitions that produce two variants is extensively described but the algebraic developments for the general case are more limited.

Most of the studies on the reconstructive transitions have actually been performed in metallurgy for many different

\footnotetext{
$\mathbf{1}$ The term 'non-disruptive' relies on the assumption that some symmetry elements of the new structure (daughter phase) can be described in the frame of reference of the old one (parent phase). Guymont does not consider these transitions as reconstructive ones but we think they are, according to the definition given by Tolédano \& Dmitriev (1996).
} 
aims: (i) to determine by EBSD the exact orientation relationship in steels (Morito et al., 2003; Kitahara et al., 2005); (ii) to understand the texture evolution during different heat treatments in Ti alloys (Gey \& Humbert, 2003) or in brass (Standford \& Bate, 2005); or (iii) to correlate the fracture mechanisms of steels to their microstructures (Gourgues et al., 2000). Indeed, after the transition, the parent grains are generally hardly visible. Therefore, a key point is the reconstruction of the parent grains from the data obtained by EBSD on the daughter grains. The most advanced theoretical studies on this subject have been proposed by Humbert et al: a method to calculate the number of orientational variants for Burgers transitions is given by Humbert et al. (1992) and a method to determine the orientation of the $\beta$-parent grains is proposed by Humbert et al. (1995) and Humbert \& Gey (2002). However, these studies lack generality. The algebraic tools used for the Landau transitions (for example the coset decomposition and the Lagrange theorem) are not explicitly recognized. Moreover, some results are incomplete (see \$9.1).

\subsection{Precipitation}

Precipitates in an orientation relationship inside a matrix have been mainly studied to simulate the complex diffraction patterns obtained by TEM (Akbay et al., 1994; Cayron, 2000). General theoretical concepts have been given by Cahn \& Kalonji (1981): the orientation relationship between two crystals of phase I (parent) and phase II (variant) is explicitly introduced by the linear transformation $(R / t)$, where $R$ is the rotation that orientates crystal II with reference to crystal I and $t$ is the translation that gives the position of the lattice of crystal II with reference to the lattice of crystal I. The set of symmetry operations of the space group of crystal II, denoted $\mathbf{G}_{0}^{\mathrm{II}}$, is written in a reference coordinate system associated with crystal I:

$$
\mathbf{G}^{\mathrm{II}}=(R / t) \mathbf{G}_{0}^{\mathrm{II}}(R / t)^{-1} .
$$

This equation leads directly to the set of common symmetry operations: $\mathbf{H}=\mathbf{G}_{0}^{\mathrm{I}} \cap \mathbf{G}^{\mathrm{II}}$, where $\mathbf{H}$ is a subgroup of $\mathbf{G}_{0}^{\mathrm{I}}$ called the 'intersection group'. It also determines the precipitate morphology. With arguments similar to those given for the Landau transitions, the variants of crystals II in matrix I appear from the left coset decomposition of $\mathbf{H}$ into $\mathbf{G}_{0}^{\mathrm{I}}$, and their number is given, here again, by the Lagrange formula $N=\left|\mathbf{G}^{\mathrm{I}}\right| /|\mathbf{H}|$. The concepts introduced by Cahn \& Kalonji (1981) go far beyond the morphologies. They have led to a general approach of grain boundaries (Gratias \& Portier, 1982; Kalonji, 1985) that unifies and clarifies the notions of coincidence site lattice (CSL), $O$-lattice and displacement shift lattice, previously introduced by Bollmann (1970, 1982). However, the orientation relationships between the variants are not studied and the algebraic tools (orbits, stabilizers) are not used as is done for the Landau transitions.

\subsection{Twinning}

Twinning has been studied for more than a hundred years (Friedel, 1904) and nowadays is often treated as a phase transition (by deformation or recrystallization). Thus, one can be tempted to use the algebraic notions reported in $\$ 2.1$, but one must be very cautious because all the concepts developed for the Landau transitions cannot be directly applied for twinning. In Hahn \& Klapper (2003), K, the so-called 'composite symmetry group' is introduced from $\mathbf{H}$, the point group of an untwinned crystal, and $k$, a twin law, by a coset decomposition similar to equation (1). $\mathbf{K}$ is believed to be a supergroup of $\mathbf{H}$ (i.e. $\mathbf{H}$ is a subgroup of $\mathbf{K}$, as is the case for the Landau transitions); however, in general, the composite 'crystal', strictly speaking, is not a crystal (no point group can be attributed) and the operations between the 'partners' (i.e. the variants that constitute this composite structure) are only partial operations. Thus, $\mathbf{K}$ is in general not a group. A rigorous approach to twinning by applying some concepts of reconstructive transitions (in terms of the non-disruption condition) and by using the intersection group presented in equation (2) is given by Wadhawan (1997).

We present now our own approach on the subject. It includes and unifies all the notions presented before (but of course not all the particular developments of each case). Comparisons with past studies are discussed in the Remarks at the end of each section. The important aim of the present paper is to show that the algebraic structure constituted by the variants (created by phase transition, precipitation or twinning) is not a group but a groupoid.

\section{Crystals oriented in space, internal symmetries}

The term 'phase' usually designates a class of materials with particular physical or chemical properties. In crystallography, for solid materials, it is restricted to particular types of atomic bonding and, if the materials are crystalline, the term also refers to the atomic arrangements, i.e. the atomic positional symmetry (space-group type) and the metric (metric tensor). The term 'crystal' designates a solid constituted by a crystalline phase and orientated in space. In this paper, phases will be denoted by a Greek character $(\beta)$ and crystals will also be denoted by a Greek character $(\beta)$ with an index when required $\left(\beta_{i}\right)$. From now on, we consider a crystal only by its orientation (and not by its position) in a fixed reference coordinate system. A crystal is then defined by (i) its internal orientational symmetries (one of the 32 point groups) and (ii) its orientation in space. We point out that a crystal is more than a simple oriented lattice (the point group would be reduced to the holohedry group). The orientation of a crystal is usually given by three Euler angles; see for example (Kocks et al., 1998) for the numerous conventions of Euler angles. However, many different triplets of Euler angles may express the same orientation because of the internal symmetries. In the present study, we choose another way to define the orientation of a crystal. A crystal $\beta$ will be defined by the set of its equivalent bases. Two crystals are 'distinct' if their sets of equivalent bases are disjoint and 'in coincidence' (or 'equal') if their sets are equal. With this definition, a crystal appears as an equivalence class on the set of bases (the equivalence classes are defined in Appendix $A$ ). If a unique reference coordinate 
system is used, the geometrical notion of 'basis' can be transformed into a useful algebraic tool, the transformation matrix.

\subsection{Transformation matrices}

Let us consider two bases $\mathrm{B}_{1}$ and $\mathrm{B}_{2}$. The basis $\mathrm{B}_{2}$ is formed by three non-collinear vectors trivially expressed by

$$
\mathbf{a}_{/ \mathrm{B}_{2}}^{\mathrm{B}_{2}}=\left[\begin{array}{l}
1 \\
0 \\
0
\end{array}\right], \quad \mathbf{b}_{/ \mathrm{B}_{2}}^{\mathrm{B}_{2}}=\left[\begin{array}{l}
0 \\
1 \\
0
\end{array}\right], \quad \mathbf{c}_{/ \mathrm{B}_{2}}^{\mathrm{B}_{2}}=\left[\begin{array}{l}
0 \\
0 \\
1
\end{array}\right] .
$$

The lower index (with /) represents the basis in which the vector is written and the upper index represents the basis constituted by the vector. These vectors can also be expressed in $\mathrm{B}_{1}$ and they become:

$$
\mathbf{a}_{/ \mathrm{B}_{1}}^{\mathrm{B}_{2}}=\left[\begin{array}{l}
u_{1} \\
u_{2} \\
u_{3}
\end{array}\right], \quad \mathbf{b}_{/ \mathrm{B}_{1}}^{\mathrm{B}_{2}}=\left[\begin{array}{l}
v_{1} \\
v_{2} \\
v_{3}
\end{array}\right], \quad \mathbf{c}_{/ \mathrm{B}_{1}}^{\mathrm{B}_{2}}=\left[\begin{array}{l}
w_{1} \\
w_{2} \\
w_{3}
\end{array}\right] .
$$

We denote $\left[\mathrm{B}_{1} \triangleright \mathrm{B}_{2}\right]$ as the transformation matrix between the two bases $\left(B_{1}, B_{2}\right)$. It is given by

$$
\left[\mathrm{B}_{1} \triangleright B_{2}\right]=\left[\mathbf{a}_{/ \mathrm{B}_{1}}^{\mathrm{B}_{2}}, \mathbf{b}_{/ \mathrm{B}_{1}}^{\mathrm{B}_{2}}, \mathbf{c}_{/ \mathrm{B}_{1}}^{\mathrm{B}_{2}}\right]=\left[\begin{array}{ccc}
u_{1} & v_{1} & w_{1} \\
u_{2} & v_{2} & w_{2} \\
u_{3} & v_{3} & w_{3}
\end{array}\right] .
$$

Since $\left[B_{1} \triangleright B_{2}\right]\left[\mathrm{B}_{2} \triangleright \mathrm{B}_{1}\right]=E$ (the $3 \times 3$ identity matrix), it follows that $\left[\mathrm{B}_{1} \triangleright \mathrm{B}_{2}\right]^{-1}=\left[\mathrm{B}_{2} \triangleright \mathrm{B}_{1}\right]$. Moreover, for any intermediate basis $\mathrm{B}_{0}$ : $\left[\mathrm{B}_{1} \triangleright \mathrm{B}_{2}\right]=\left[\mathrm{B}_{1} \triangleright \mathrm{B}_{0}\right]\left[\mathrm{B}_{0} \triangleright \mathrm{B}_{2}\right]$. If $f$ is a linear function expressed in a basis $\mathrm{B}_{1}$ by the matrix $\mathrm{F}, f$ is expressed in a basis $\mathrm{B}_{2}$ by the matrix $\left[\mathrm{B}_{1} \triangleright \mathrm{B}_{2}\right]^{-1} \mathrm{~F}\left[\mathrm{~B}_{1} \triangleright \mathrm{B}_{2}\right]$. One must be cautious when interpreting transformation matrices in terms of linear functions. ${ }^{2}$

\subsection{Orientational symmetries expressed as transformation matrices, composition rule}

We consider a crystal $\beta$ with the set of its equivalent bases denoted $\mathbf{B}^{\beta}=\left\{\mathbf{B}_{i}^{\beta}\right\}$. Let us arbitrarily choose one of the possible bases $\mathbf{B}_{1}^{\beta}=\left(\mathbf{a}^{\beta}, \mathbf{b}^{\beta}, \mathbf{c}^{\beta}\right) \in \mathbf{B}_{\beta}$ as a fixed reference coordinate system. The bases $\mathrm{B}_{i}^{\beta}$ of $\mathbf{B}^{\beta}$ are given by the transformation matrices $\left[\mathrm{B}_{1}^{\beta} \triangleright \mathrm{B}_{i}^{\beta}\right]=g_{i}^{\beta}$, where $g_{i}^{\beta}$ is an orientational symmetry of the crystal $\beta$. We denote as $\mathbf{G}^{\beta}=\left\{g_{i}^{\beta}\right\}$ the set of all the symmetry operations ${ }^{3}$, i.e. the point group of the crystal $\beta$. If another basis of the crystal $\beta$ is chosen as a new reference coordinate system, for example $\mathrm{B}_{n}^{\beta} \in \mathbf{B}^{\beta}$ instead of $\mathrm{B}_{1}^{\beta}$, then $g_{i}^{\beta}$ becomes in this system $\left(g_{i}^{\beta}\right)^{\prime}=$

\footnotetext{
${ }^{2}$ Clearly, if we denote by $f$ and $g$ the linear functions associated respectively with the matrices $\mathrm{F}$ and $\mathrm{G}$ expressed in the same coordinate system $\mathrm{B}_{0}$, their composition $f \circ g$ ('first $g$ then $f$ ') is expressed by the product $\mathrm{FG}$ in $\mathrm{B}_{0}$. However, if we denote by $f$ and $g$ the linear functions associated with the matrices $F=\left[B_{0} \triangleright B_{1}\right]$ and $G=\left[B_{1} \triangleright B_{2}\right]$, it must be noted that these matrices are expressed in different coordinate systems. We can write them in the same system $\mathrm{B}_{0}$; they become $\mathrm{F}$ and $\mathrm{FGF}^{-1}$, respectively, and now it is the composition $g \circ f$ ('first $f$ then $g$ ') that is expressed by FG in $\mathrm{B}_{0}$. We conclude that the notation $f g(=g \circ f)$ is more appropriate to write the composition of functions associated with transformation matrices.

${ }^{3}$ Sometimes the term 'symmetry operators' is used, but we will keep this term for another mathematical concept, i.e. a class of equivalence of symmetry operations (see $\S 6.2$ ).
}

$\left[\mathrm{B}_{1}^{\beta} \triangleright \mathrm{B}_{n}^{\beta}\right]^{-1} g_{i}^{\beta}\left[\mathrm{B}_{1}^{\beta} \triangleright \mathrm{B}_{n}^{\beta}\right]=\left(g_{n}^{\beta}\right)^{-1} g_{i}^{\beta} g_{n}^{\beta}$, the conjugate of $g_{i}^{\beta}$ by $g_{n}^{\beta}$. Since a group acts on itself by conjugation $\left(\mathbf{G}^{\beta}\right)^{\prime}=\mathbf{G}^{\beta}$, we conclude that $\mathbf{G}^{\beta}$ does not depend on the choice of the reference basis if this one belongs to $\mathbf{B}^{\beta}$. In this way, the crystal is 'self-referred' and $\mathbf{G}^{\beta}$ constitutes its internal symmetries. There is no distinction between the point group of a phase $\beta$ and the point group of a $\beta$ crystal if this one is self-referred.

In order to reduce the length of equations that will be developed in the following, we note:

$$
g_{i k}^{\beta}=\left[\mathrm{B}_{i}^{\beta} \triangleright \mathrm{B}_{k}^{\beta}\right]=\left(g_{i}^{\beta}\right)^{-1} g_{k}^{\beta} .
$$

The fact that the matrices $g_{i}^{\beta}$ are implicitly expressed in the basis $\mathrm{B}_{1}^{\beta}$ appears in $g_{1}^{\beta}=E$ and $g_{k}^{\beta}=g_{1 k}^{\beta}$. One may check that the matrices $g_{i k}^{\beta}$ can be composed according to the following rule:

$$
\begin{gathered}
g_{i k}^{\beta} g_{k l}^{\beta}=g_{i l}^{\beta} \\
\left(g_{i k}^{\beta}\right)^{-1}=g_{k i}^{\beta} .
\end{gathered}
$$

The composition (6) has a mathematical meaning: the indexes $i, k$ and $l$ follow the composition rule of the trivial pair groupoid: two pairs $(i, k)$ and $\left(k^{\prime}, l\right)$ can be composed if and only if $k=k^{\prime}$ and then $(i, k)\left(k^{\prime}, l\right)=(i, l)$ as developed in $\S 7.1$. We compare our notation with the one chosen by Janovec. ${ }^{4}$

\subsection{Internal classes of transformation matrices}

Now, let us consider a crystal $\alpha$ oriented in space, and $\mathrm{B}_{0}$ an arbitrary fixed reference coordinate system. One can orientate this crystal with the help of any transformation matrix from $\mathrm{B}_{0}$ to one of the crystal bases. In other words, the crystal $\alpha$ is oriented by any matrix of the set $\left\{\left[\mathrm{B}_{0} \triangleright \mathrm{B}_{n}^{\alpha}\right], \mathrm{B}_{n}^{\alpha} \in \mathbf{B}^{\alpha}\right\}$. Each orientation matrix of this set can be decomposed according to: $\left[\mathrm{B}_{0} \triangleright \mathrm{B}_{n}^{\alpha}\right]=\left[\mathrm{B}_{0} \triangleright \mathrm{B}_{1}^{\alpha}\right]\left[\mathrm{B}_{1}^{\alpha} \triangleright \mathrm{B}_{n}^{\alpha}\right]=\mathrm{T} g_{n}^{\alpha}$, with $\mathrm{T}=\left[\mathrm{B}_{0} \triangleright \mathrm{B}_{1}^{\alpha}\right]$. Hence, the set of all the equivalent transformation (or orientation) matrices of a crystal $\alpha$ becomes

$$
\left\{\mathrm{Tg}_{n}^{\alpha}, g_{n}^{\alpha} \in \mathbf{G}^{\alpha}\right\}=\mathrm{TG}^{\alpha} .
$$

We can then introduce an 'internal' equivalence relation, denoted $\sim_{I}^{\alpha}$ (with $I$ for internal), on the matrices that define (the OR of) the same crystal $\alpha$ : two transformation matrices $\mathrm{T}_{k}$ and $\mathrm{T}_{l}$ define the same crystal $\alpha$ if and only if $\exists\left(g_{k}^{\alpha}, g_{l}^{\alpha}\right) \in$ $\left(\mathbf{G}^{\alpha}\right)^{2}$ and $\exists \mathrm{T}$ an invertible matrix such that $\mathrm{T}_{k}=\mathrm{Tg}_{k}^{\alpha}$ and $\mathrm{T}_{l}=$ $\mathrm{T} g_{l}^{\alpha}$. This condition can be written without explicit reference to $\mathrm{T}$ (or to the fixed reference coordinate system $\mathrm{B}_{0}$ ) by noticing that $\mathrm{T}_{k}^{-1} \mathrm{~T}_{l}=\left(g_{k}^{\alpha}\right)^{-1} g_{l}^{\alpha}=g_{k l}^{\alpha}$. It becomes

$$
\mathrm{T}_{k} \sim_{I}^{\beta} \mathrm{T}_{l} \Longleftrightarrow \mathrm{T}_{k}^{-1} \mathrm{~T}_{l} \in \mathbf{G}^{\alpha}
$$

$\mathrm{TG}^{\alpha}$, the set of matrices of the orientation of the crystal $\alpha$, does not form a group. However, since the 'left class of equivalence' found in group theory (Appendix $A$ ) is similar to condition (9), we will also use the same term for (9). One may

\footnotetext{
${ }^{4}$ The notation introduced in equation (5) slightly differs from the one used by Janovec \& Přívratská (2003): we use $g_{k i}$ instead of Janovec's $g_{i k}$. Actually, Janovec notes the 'switching operation' that transforms the domain state $\mathbf{S}_{i}$ into $\mathbf{S}_{k}: \mathbf{S}_{k}=g_{i k} \mathbf{S}_{i}$. With our notation (5), we write $\mathbf{S}_{k}=g_{k i} \mathbf{S}_{i}$ and the composition rule (6) becomes: if $\mathbf{S}_{k}=g_{k i} \mathbf{S}_{i}$ and $\mathbf{S}_{l}=g_{l k} \mathbf{S}_{k}$, then $\mathbf{S}_{l}=g_{l i} \mathbf{S}_{i}$ with $g_{l i}=g_{l k} g_{k i}$. The reader may see that this composition is more intuitive than with the Janovec notation.
} 
also notice that $\mathbf{G}^{\alpha}$ acts trivially transitively at the right of the set $\mathrm{TG}^{\alpha}$ (Appendix $B$ ).

\section{Orientation relationships and external symmetries}

\subsection{Matrix expression of an orientation relationship}

We denote by $\beta$ a crystal with a fixed orientation and by $\alpha_{i}$ the crystals of phase $\alpha$ in equivalent orientation relationships (OR) with $\beta$. The crystals $\alpha_{i}$ are generated by a $\beta \rightarrow \alpha$ structural phase transition, by twinning or by precipitation. We denote as $\mathbf{G}^{\alpha}=\left\{g_{i}^{\alpha}\right\}$ the point group of the phase $\alpha$. At each crystal $\alpha_{i}$ can be associated the set of equivalent bases $\mathbf{B}^{\alpha_{i}}=$ $\left\{\mathrm{B}_{n}^{\alpha_{i}}\right\}$ such that $\left\{\left[\mathrm{B}_{1}^{\alpha_{i}} \triangleright \mathrm{B}_{n}^{\alpha_{i}}\right]\right\}=\mathbf{G}^{\alpha}$.

In order to compare a crystal $\alpha_{i}$ to another crystal $\alpha_{j}$, a unique reference coordinate system must be chosen, for example $\mathrm{B}_{1}^{\beta}$. The OR between the variant $\alpha_{1}$ and the parent crystal $\beta$ is given by the transformation matrix $\left[\mathrm{B}_{1}^{\beta} \triangleright \mathrm{B}_{1}^{\alpha_{1}}\right]$ which will be denoted $T_{>}$. The subscript $>$represents the direction of the transition (see $\$ 5.2$ ).

$\mathrm{T}_{>}$can be explicitly calculated from the usual form of an OR given by two couples of parallel and similarly oriented vectors expressed in their own crystal lattice: $\mathbf{u}^{\alpha_{1}} \| \mathbf{u}^{\beta}$ and $\mathbf{v}^{\alpha_{1}} \| \mathbf{v}^{\beta}$ with $\mathbf{u}^{\alpha_{1}}$ and $\mathbf{v}^{\alpha_{1}}$ not parallel (see example in Table 1). A third couple of vectors $\mathbf{w}^{\alpha_{1}} \| \mathbf{w}^{\beta}$ can then be created with $\mathbf{w}^{\alpha_{1}}=$ $\mathbf{u}^{\alpha_{1}} \wedge \mathbf{v}^{\alpha_{1}}$ and $\mathbf{w}^{\beta}=\mathbf{u}^{\beta} \wedge \mathbf{v}^{\beta}$ (the calculations of the cross product are not reported here, they imply expression of the vectors in an orthogonal coordinate system with the help of the structure tensors). Now, a new basis $\mathrm{B}_{c}$ common to both crystals $\alpha_{1}$ and $\beta$ is introduced and defined by

$$
\left[\mathrm{B}_{1}^{\alpha_{1}} \triangleright B_{c}\right]=\left[\mathbf{u}^{\alpha_{1}} /\left\|\mathbf{u}^{\alpha_{1}}\right\|, \mathbf{v}^{\alpha_{1}} /\left\|\mathbf{v}^{\alpha_{1}}\right\|, \mathbf{w}^{\alpha_{1}} /\left\|\mathbf{w}^{\alpha_{1}}\right\|\right]
$$

and

$$
\left[\mathrm{B}_{1}^{\beta} \triangleright B_{c}\right]=\left[\mathbf{u}^{\beta} /\left\|\mathbf{u}^{\beta}\right\|, \mathbf{v}^{\beta} /\left\|\mathbf{v}^{\beta}\right\|, \mathbf{w}^{\beta} /\left\|\mathbf{w}^{\beta}\right\|\right]
$$

Then, $\mathrm{T}_{>}$is explicitly deduced from the OR by

$$
\mathrm{T}_{>}=\left[\mathrm{B}_{1}^{\beta} \triangleright \mathrm{B}_{1}^{\alpha_{1}}\right]=\left[\mathrm{B}_{1}^{\beta} \triangleright \mathrm{B}_{c}\right]\left[\mathrm{B}_{1}^{\alpha_{1}} \triangleright \mathrm{B}_{c}\right]^{-1} .
$$

We point out that the directions of the 'parallel and similarly oriented' vectors are important if the crystals are not centrosymmetric.

Since equation (10) gives the OR of the first variant, we write

$$
\mathrm{T}^{\beta \rightarrow \alpha_{1}}=\mathrm{T}_{>}
$$

\subsection{External classes of transformation matrices}

The parent crystal $\beta$ has its own internal symmetries, which, in general, do not correspond to the internal symmetries of the crystals $\alpha_{i}$. Using the groupoid formalism (Weinstein, 1996), we will say that the symmetries of the crystal $\beta$ act as 'external' symmetries for the crystals $\alpha_{i}$. We now choose the following indexing rule for the crystals $\alpha_{i}$ :

$$
\left[\mathrm{B}_{i}^{\beta} \triangleright \mathrm{B}_{1}^{\alpha_{i}}\right]=\left[\mathrm{B}_{1}^{\beta} \triangleright \mathrm{B}_{1}^{\alpha_{1}}\right]=\mathrm{T}_{>} .
$$

Table 1

Orientation relationship (OR) with a Burgers transition $\beta \rightarrow \alpha$.

$$
\begin{aligned}
& \text { Burgers transformation } \\
& \text { Parent }=\beta=\text { body-centered cubic (b.c.c.): } \mathbf{G}^{\beta}=m 3 m \\
& \text { Daughter }=\alpha=\text { hexagonal close packed (h.c.p.): } \mathbf{G}^{\alpha}=6 / \mathrm{mmm}
\end{aligned}
$$

$(001)_{\alpha} \|(110)_{\beta}$

$[100]_{\alpha} \|[1 \overline{1} 1]_{\beta}$

Then, the matrix that defines the orientation of a crystal $\alpha_{i}$ is given by $\left[\mathrm{B}_{1}^{\beta} \triangleright \mathrm{B}_{1}^{\alpha_{i}}\right]=\left[\mathrm{B}_{1}^{\beta} \triangleright \mathrm{B}_{i}^{\beta}\right]\left[\mathrm{B}_{i}^{\beta} \triangleright \mathrm{B}_{1}^{\alpha_{i}}\right]=g_{i}^{\beta} \mathrm{T}_{>}$. One may then write

$$
\mathrm{T}^{\beta \rightarrow \alpha_{i}}=g_{i}^{\beta} \mathrm{T}_{>}
$$

The set of the transformation matrices $\mathrm{T}^{\beta \rightarrow \alpha_{i}}$ of all the crystals $\alpha_{i}$ inherited from the same parent crystal $\beta$ is

$$
\left\{g_{i}^{\beta} \mathrm{T}_{>}, g_{i}^{\beta} \in \mathbf{G}^{\beta}\right\}=\mathbf{G}^{\beta} \mathbf{T}_{>} .
$$

For example, it means that for an $m 3 m$ parent crystal the number of daughter crystals is at maximum equal to $\left|\mathbf{G}^{\beta}\right|=48$. However, some of the daughter crystals are in coincidence and we will see in the next section that their number is actually a divisor of $\left|\mathbf{G}^{\beta}\right|$.

We can then introduce an 'external' equivalence relation, denoted $\sim_{E}^{\beta}$ (with $E$ for external), on the matrices that define (the OR of) the crystals inherited from the same parent crystal: two transformation matrices $\mathrm{T}_{k}$ and $\mathrm{T}_{l}$ define two daughter crystals $\alpha_{k}$ and $\alpha_{l}$ if and only if $\exists\left(g_{k}^{\beta}, g_{l}^{\beta}\right) \in\left(\mathbf{G}^{\beta}\right)^{2}$ and $\exists \mathrm{T}_{>}$an invertible matrix such that $\mathrm{T}_{k}=g_{k}^{\beta} \mathrm{T}_{>}$and $\mathrm{T}_{l}=$ $g_{l}^{\beta} \mathrm{T}_{>}$. This condition can be written without explicit reference to $\mathrm{T}_{>}$by noticing that $\mathrm{T}_{k} \mathrm{~T}_{l}^{-1}=g_{k}^{\beta}\left(g_{l}^{\beta}\right)^{-1}$. The condition becomes

$$
\mathrm{T}_{k} \sim_{E}^{\beta} \mathrm{T}_{l} \Longleftrightarrow \mathrm{T}_{k} \mathrm{~T}_{l}^{-1} \in \mathbf{G}^{\beta}
$$

$\mathbf{G}^{\beta} \mathrm{T}_{>}$, the set of orientations of the crystals $\alpha_{i}$, does not form a group. However, since the 'right class of equivalence' (Appendix $A$ ) is similar to condition (15), we will also use the same term for (15). One may also notice that $\mathbf{G}^{\beta}$ acts trivially transitively at the left of the set $\mathbf{G}^{\beta} \mathrm{T}_{>}$(Appendix $B$ ).

Remark 1. If the two phases $\alpha$ and $\beta$ are the same, the crystals $\beta$ and $\alpha$ are called twinned 'partners' and, if the condition of twin orientation relationship is obeyed (the two crystals have a common lattice row and a parallel lattice plane), the transformation matrix $\mathrm{T}^{\beta \rightarrow \alpha}$ of equation (11) corresponds to the 'twin law' (Hahn \& Klapper, 2003). With cubic crystals, a twin law is given by a rotation matrix with rational elements $R=\frac{1}{\Sigma} a_{i j}$, such that $a_{i j}$ are integers with no integral factor (Warrington \& Bufalini, 1971) and $\Sigma$ is the ratio of the unit volume of the CSL lattice referred to the unit volume of the crystal lattice (Grimmer et al., 1974). The present approach generalizes the twin law or the CSL lattice because no restriction exists on the vectors $\mathbf{u}, \mathbf{v}, \mathbf{w}$ introduced to define $\mathrm{T}_{>}$; they can have real coordinates. 


\section{Algebraic definition of the orientational variants}

\subsection{The set of variants}

We consider two crystals $\alpha_{i}$ and $\alpha_{j}$ that are variants inherited from the same parent crystal $\beta$, with the associated externally equivalent transformation matrices $\mathrm{T}^{\beta \rightarrow \alpha_{i}}=g_{i}^{\beta} \mathrm{T}_{>}$and $\mathrm{T}^{\beta \rightarrow \alpha_{j}}=$ $g_{j}^{\beta} \mathrm{T}_{>}$. These two matrices define the orientation of the same crystal (i.e. the crystals are in coincidence, ' $\alpha_{i}=\alpha_{j}$ ') if and only if they are internally equivalent [see equation (9)]:

$$
\mathrm{T}^{\beta \rightarrow \alpha_{i}} \stackrel{\alpha}{=} \mathrm{T}_{I}^{\beta \rightarrow \alpha_{j}} \Leftrightarrow\left(\mathrm{T}^{\beta \rightarrow \alpha_{i}}\right)^{-1} \mathrm{~T}^{\beta \rightarrow \alpha_{j}} \in \mathbf{G}^{\alpha}
$$

$\Leftrightarrow\left(g_{i}^{\beta}\right)^{-1} g_{j}^{\beta}=g_{i j}^{\beta} \in T_{>} \mathbf{G}^{\alpha} T_{>}^{-1}$. Since $g_{i j}^{\beta}$ is also an element of $\mathbf{G}^{\beta}$, the condition becomes

$$
\mathrm{T}^{\beta \rightarrow \alpha_{i}} \stackrel{\alpha}{=} \mathrm{T}^{\beta \rightarrow \alpha_{j}} \Leftrightarrow g_{i j}^{\beta} \in \mathbf{G}^{\beta} \cap \mathrm{T}_{>} \mathbf{G}^{\alpha} \mathrm{T}_{>}^{-1} .
$$

It must be remembered that $\mathrm{T}_{>}=\left[\mathrm{B}_{1}^{\beta} \triangleright \mathrm{B}_{1}^{\alpha_{1}}\right]$; therefore, in equation (16), $\mathrm{T}_{>} \mathbf{G}^{\alpha} \mathrm{T}_{>}^{-1}$ constitutes the set of symmetry matrices of the crystal $\alpha_{1}$ expressed in the basis $\mathrm{B}_{1}^{\beta}$ of the crystal $\beta$. As the mapping

$$
\psi: g \rightarrow \mathrm{T}_{>} g \mathrm{~T}_{>}^{-1}
$$

is an isomorphism, $\mathrm{T}_{>} \mathbf{G}^{\alpha} \mathrm{T}_{>}^{-1}=\psi\left(\mathbf{G}^{\alpha}\right)$ is also a group. Since the intersection of two groups is not always a group, the reader is invited to verify that the elements of $\mathbf{G}^{\beta} \cap \mathrm{T}_{>} \mathbf{G}^{\alpha} \mathrm{T}_{>}^{-1}$ effectively form a group that is a subgroup of $\mathbf{G}^{\beta}$. We denote this subgroup $\mathbf{H}^{\beta}$ and call it the intersection group as done by Cahn \& Kalonji (1981). More precisely, $\mathbf{H}^{\beta}$ can also be written $\mathbf{H}^{\beta / \alpha_{1}}$ because $\mathbf{H}^{\beta}$ depends on $\mathrm{T}_{>}$, which is a function of the two bases $\mathrm{B}_{1}^{\beta}$ and $\mathrm{B}_{1}^{\alpha_{1}}$ (the sign / just means 'with reference to'). $\mathbf{H}^{\beta}$ is the set of symmetries common to crystal $\beta$ and crystal $\alpha_{1}$. We recall here that the external morphology of precipitates embedded in a matrix has the symmetries of the $\mathbf{H}^{\beta}$ group (Cahn \& Kalonji, 1981).

To sum up, two external transformations $\mathrm{T}^{\beta \rightarrow \alpha_{i}}$ and $\mathrm{T}^{\beta \rightarrow \alpha_{j}}$ (elements of $\mathbf{G}^{\beta} \mathrm{T}_{>}$) point to the same daughter crystal (' $\alpha_{i}=$ $\alpha_{j}^{\prime}$ ) if and only if they are also elements of $\mathrm{T}_{>} \mathbf{G}^{\alpha}$, i.e. if and only if $g_{i j}^{\beta}$ is an element of

$$
\mathbf{H}^{\beta}=\mathbf{H}^{\beta / \alpha_{1}}=\mathbf{G}^{\beta} \cap \mathrm{T}_{>} \mathbf{G}^{\alpha} \mathbf{T}_{>}^{-1} \leq \mathbf{G}^{\beta} .
$$

The reader may now check that the transformation matrices giving equivalent ORs of the crystal $\alpha_{i}$ can be deduced from the matrix presented in equation (13); they form the set

$$
\mathbf{T}^{\beta \rightarrow \alpha_{i}}=g_{i}^{\beta} \mathbf{H}^{\beta} \mathbf{T}_{>} .
$$

Reciprocally, two crystals $\alpha_{i}$ and $\alpha_{j}$ are distinct if and only if $g_{i}^{\beta} \mathbf{H}^{\beta}$ and $g_{j}^{\beta} \mathbf{H}^{\beta}$ are distinct sets. We denote by $g_{i}^{\beta} \mathbf{H}^{\beta}$ each left $\mathbf{H}^{\beta}$ coset in $\mathbf{G}^{\beta}$. The canonical decomposition of $\mathbf{G}^{\beta}$ into left cosets leads to

$$
\mathbf{G}^{\beta}=g_{1}^{\beta} \mathbf{H}^{\beta} \cup g_{2}^{\beta} \mathbf{H}^{\beta} \cup \ldots \cup g_{\mathrm{N}^{\alpha}}^{\beta} \mathbf{H}^{\beta},
$$

where $g_{1}^{\beta}=E$. Now, each coset is treated as a unique mathematical entity. Their set (i.e. a set of sets) is called the quotient set; it is denoted $\mathbf{G}^{\beta} / \mathbf{H}^{\beta}$ :

$$
\mathbf{G}^{\beta} / \mathbf{H}^{\beta}=\left\{g_{1}^{\beta} \mathbf{H}^{\beta}, g_{2}^{\beta} \mathbf{H}^{\beta}, \ldots, g_{\mathrm{N}^{\alpha}}^{\beta} \mathbf{H}^{\beta}\right\} .
$$

We have proved that each orientation of a crystal $\alpha_{i}$ (each orientational crystal) is defined by one left $\mathbf{H}^{\beta}$ coset, i.e. an element of $\mathbf{G}^{\beta} / \mathbf{H}^{\beta}$. In all the following, we will associate with the crystal $\alpha_{i}$ (geometric notion) a left coset (algebraic notion) by directly writing

$$
\alpha_{i}=g_{i}^{\beta} \mathbf{H}^{\beta} .
$$

The distinct $\alpha_{i}$ will be called orientational variants. Their number $\mathrm{N}^{\alpha}$ in the decomposition (20) is given by the Lagrange formula

$$
\mathbf{N}^{\alpha}=\left|\mathbf{G}^{\beta} / \mathbf{H}^{\beta}\right|=\left|\mathbf{G}^{\beta}\right| /\left|\mathbf{H}^{\beta}\right| .
$$

In the following, we will simply denote $\mathbf{V}^{\alpha}$ as the set of the $\alpha_{i}$ variants:

$$
\mathbf{V}^{\alpha}=\alpha_{i}=\mathbf{G}^{\beta} / \mathbf{H}^{\beta} .
$$

In this paper, we will consider that $\mathbf{G}^{\beta}$ acts on $\mathbf{V}^{\alpha}$ by classical left product: for any $g \in \mathbf{G}^{\beta}$ and $\alpha_{i} \in \mathbf{V}^{\alpha}, g \alpha_{i}=g\left(g_{i}^{\beta} \mathbf{H}^{\beta}\right)=$ $\left(g g_{i}^{\beta}\right) \mathbf{H}^{\beta}$. For this action, ${ }^{5}$ the stabilizer of a variant $\alpha_{i}$ is defined by

$$
\mathbf{S t a b}_{\mathbf{G}^{\beta}}\left(\alpha_{i}\right)=\left\{g^{\beta} \in \mathbf{G}^{\beta}, g^{\beta} \alpha_{i}=\alpha_{i}\right\} .
$$

It forms a subgroup of $\mathbf{G}^{\beta}$ also called the isotropy group of $\alpha_{i}$. The stabilizer of $\alpha_{1}$ is $\mathbf{S t a b}_{\mathbf{G}^{\beta}}\left(\alpha_{1}\right)=\mathbf{H}^{\beta}=\mathbf{H}^{\beta / \alpha_{1}}$. The stabilizer of each variant $\alpha_{i}$ is the intersection group of $\alpha_{i}$; it is given by

$$
\mathbf{S t a b}_{\mathbf{G}^{\beta}}\left(\alpha_{i}\right)=\mathbf{H}^{\beta / \alpha_{i}}=\mathrm{g}_{i}^{\beta} \mathbf{H}^{\beta}\left(\mathrm{g}_{i}^{\beta}\right)^{-1} .
$$

$\mathrm{g}_{i}^{\beta} \mathbf{H}^{\beta}\left(\mathrm{g}_{i}^{\beta}\right)^{-1}$ is the conjugate of $\mathbf{H}^{\beta}$ by $\mathrm{g}_{i}^{\beta}$. One may verify that it does not depend on the choice of the representative $\mathrm{g}_{i}^{\beta}$ in the coset $\alpha_{i}$ and that equation (26) can be directly written

$$
\mathbf{H}^{\beta / \alpha_{i}}=\alpha_{i} \alpha_{i}^{-1} \text {. }
$$

$\mathbf{H}^{\beta / \alpha_{i}}$ then appears as the product of two sets of matrices. ${ }^{6}$

The present approach can be considered as a generalization of the previous studies of phase transition and twinning (see $\S 2)$ :

(a) Landau transitions correspond to the case $\mathbf{G}^{\alpha} \leq \mathbf{G}^{\beta}$, then $\mathbf{H}^{\beta}=\mathbf{G}^{\alpha}$ and $\mathrm{N}^{\alpha}=\left|\mathbf{G}^{\beta}\right| /\left|\mathbf{G}^{\alpha}\right|$ as already proved by Janovec (1972) and Van Tendeloo \& Amelinckx (1974).

(b) Twinning transitions correspond to the case $\mathrm{T}_{>} \neq E$ and $\mathbf{G}^{\beta}=\mathbf{G}^{\alpha}$, then $\mathbf{H}^{\beta} \neq \mathbf{G}^{\beta}$ (for twinning with $\Sigma \neq 1$ ). In the case of cubic materials with $\Sigma=3$, we find four twinning variants. Their orientation matrices $\mathbf{T}^{\beta \rightarrow \alpha_{i}}$ have already been found by Gottstein (1984). More details will be given by Cayron (2006).

It is of primary importance to note that $\mathbf{V}^{\alpha}=\mathbf{G}^{\beta} / \mathbf{H}^{\beta}$ is a quotient set and, in consequence, does not have a group structure in general. $\mathbf{V}^{\alpha}$ can be considered as a group if and only if $\mathbf{H}^{\beta}$ is a normal subgroup of $\mathbf{G}^{\beta}$ (denoted $\mathbf{H}^{\beta} \triangleleft \mathbf{G}^{\beta}$, see Appendix A). Only in this case can $\mathbf{V}^{\alpha}$ be called a 'supergroup' or 'composite group'. Moreover,

$$
\mathbf{H}^{\beta} \triangleleft \mathbf{G}^{\beta} \Rightarrow \forall \alpha_{i} \in \mathbf{V}^{\alpha}, \operatorname{Stab}_{\mathbf{G}^{\beta}}\left(\alpha_{i}\right)=\mathbf{H}^{\beta},
$$

\footnotetext{
${ }^{5}$ One must be very careful not to be confused by the type of action used by the author. For example, Janovec usually uses the conjugate action of $\mathbf{G}^{\beta}$ on its subgroups $\mathbf{H}$ defined by $g . \mathbf{H}=g \mathbf{H} g^{-1}$. He uses $\mathbf{I}_{\mathbf{g}}(x)$ for $\mathbf{S t a b}_{\mathbf{G}^{\beta}}^{c}(x)$ and, as detailed in Appendix $B$, for this action, $\mathbf{S t a b}_{\mathbf{G}^{\beta}}^{c}\left(\mathbf{H}^{\beta}\right)=N_{g}\left(\mathbf{H}^{\beta}\right)$ the normalizer of $\mathbf{H}^{\beta}$.

${ }^{6}$ We recall that the product of two sets $\mathbf{X}$ and $\mathbf{Y}$ is $\mathbf{X Y}=\left\{x_{i} y_{j}, x_{i} \in \mathbf{X}\right.$ and $y_{j} \in$ $\mathbf{Y}\}$ and that $\mathbf{X}^{-1}=\left\{x_{i}^{-1}, x_{i} \in \mathbf{X}\right\}$.
} 
which means that all variants have the same intersection group $\mathbf{H}^{\beta}$. There is a special case that implies $\mathbf{H}^{\beta} \triangleleft \mathbf{G}^{\beta}$ : when there are only two left cosets in the decomposition (20), i.e. when there are only two variants; see Janovec et al. (2003, p. 384) and Guymont (1981).

The two-dimensional example proposed in Fig. 1 is very illustrative. The point group of the parent crystal is $\mathbf{G}^{\beta}=$ $\left\{E, I, m_{x}^{\beta}, m_{y}^{\beta}, m_{x y}^{\beta}, m_{x \bar{y}}^{\beta}, r_{+\pi / 2}^{\beta}, r_{-\pi / 2}^{\beta}\right\}$, where, for instance, $m_{x}^{\beta}$ is the matrix of the mirror symmetry through the $x$ axis and $r_{+\pi / 2}^{\beta}$ the matrix of the plane rotation of angle $+\pi / 2$. All matrices of $\mathbf{G}^{\beta}$ are expressed in the same basis $\mathrm{B}_{1}^{\beta}$. The point group of the daughter phase is $\mathbf{G}^{\alpha}=\left\{E, m_{1}^{\alpha}, m_{2}^{\alpha}, m_{3}^{\alpha}, r_{+\pi / 3}^{\alpha}, r_{-\pi / 3}^{\alpha}\right\}$. For nearly all the orientation relationships (Fig. 1a), $\mathbf{H}^{\beta}$ is reduced to $\{E\}$ and the number of variants is $\mathbf{N}^{\alpha}=\left|\mathbf{G}^{\beta}\right|=8$. In these cases, $\mathbf{H}^{\beta}$ is trivially normal in $\mathbf{G}^{\beta}$; thus, each variant is equal to a singleton of $\mathbf{G}^{\beta}$, for example $\alpha_{1}=\{E\}, \alpha_{2}=\left\{m_{x}^{\beta}\right\}, \alpha_{3}=\left\{r_{+\pi / 2}^{\beta}\right\}$ etc. We note the trivial isomorphism $\mathrm{p}(x)=\{x\}$; the set of variants $\mathbf{V}^{\alpha}$ is isomorphic to $\mathbf{G}^{\beta}\left(\mathbf{V}^{\alpha} \cong_{\mathrm{p}} \mathbf{G}^{\beta}\right)$ and, in consequence, has a group structure.

However, there are two special cases that differ from this 'quasigeneral' case. In Fig. $1(b), \mathbf{H}^{\beta}$ increases to $\left\{E, m_{x}^{\beta}\right\}$ because $m_{x}^{\beta}$ of the crystal $\beta$ coincides with $m_{1}^{\alpha}$ of the crystal $\alpha_{1}$. In Fig. $1(c), \mathbf{H}^{\beta}$ increases to $\left\{E, m_{x y}^{\beta}\right\}$ because $m_{x y}^{\beta}$ coincides with $m_{1}^{\alpha}$. In these two cases, the number of variants is reduced to $N^{\alpha}=4$. Each variant is described by a couple of symmetry operations:

$$
\begin{aligned}
& \alpha_{1}=\left\{E, m_{x}^{\beta}\right\} \\
& \alpha_{2}=r_{+\pi / 2}^{\beta} \alpha_{1}=m_{x y}^{\beta} \alpha_{1}=\left\{m_{x y}^{\beta}, r_{+\pi / 2}^{\beta}\right\} \\
& \alpha_{3}=I \alpha_{1}=m_{y}^{\beta} \alpha_{1}=\left\{I, m_{y}^{\beta}\right\} \\
& \alpha_{4}=r_{-\pi / 2}^{\beta} \alpha_{1}=m_{x y}^{\beta} \alpha_{1}=\left\{r_{-\pi / 2}^{\beta}, m_{x y}^{\beta}\right\}
\end{aligned}
$$

and $\mathbf{H}^{\beta / \alpha_{1}}=\mathbf{H}^{\beta / \alpha_{3}}=\left\{E, m_{x}^{\beta}\right\}, \mathbf{H}^{\beta / \alpha_{2}}=\mathbf{H}^{\beta / \alpha_{4}}=\left\{E, m_{y}^{\beta}\right\}$. The very interesting fact is that $\mathbf{V}^{\alpha}$ is not a group any more because now $\mathbf{H}^{\beta}$ is not a normal subgroup of $\mathbf{G}^{\beta}$. For example, the reader can check in the case of Fig. $1(b)$ that $\left\{E, m_{x}^{\beta}\right\}$ and $m_{x y}^{\beta}$ do not commute. $^{7}$

Remark 2. The orientational variants $\alpha_{i}=\mathrm{g}_{i}^{\beta} \mathbf{H}^{\beta}$ are algebraically similar to the domain states $\mathbf{S}_{i}=g_{i} \mathbf{F}_{1}$ introduced in Janovec (1972) for the Landau transitions. Therefore, many of the results found by Janovec are also valid for reconstructive transitions if one takes the precaution to substitute $\mathbf{G}^{\alpha}$ by $\mathbf{H}^{\beta}$ in some parts of his equations. For example, if we call $n_{F}$ the number of distinct intersection groups (i.e. the number of distinct conjugate subgroups) and $d_{F}$ the number of variants with the same intersection group: since $\mathbf{H}^{\beta} \leq \mathrm{N}_{\mathbf{G}}\left(\mathbf{H}^{\beta}\right) \leq \mathbf{G}^{\beta}$ (see Appendix $B$ ), it follows that $n_{F}=\left|\mathbf{G}^{\beta}\right| /\left|\mathrm{N}_{\mathbf{G}}\left(\mathbf{H}^{\beta}\right)\right|$ and $d_{F}=$ $\left|\mathrm{N}_{\mathbf{G}}\left(\mathbf{H}^{\beta}\right)\right| /\left|\mathbf{H}^{\beta}\right|$ and then that $\mathrm{N}^{\alpha}=n_{F} d_{F}$.

\footnotetext{
${ }^{7}$ We recall that the product of matrices $m_{x y} m_{x}$ corresponds to the composition of linear functions $m_{x y} \circ m_{x}$ (first apply $m_{x}$ and then $m_{x y}$ ); see footnote 2. It follows that $m_{x y} m_{x}=r_{+\pi / 4}$ and $m_{x} m_{x y}=r_{-\pi / 4}$.
}

Remark 3. The equality (23) has already been proved by Humbert et al. (1992) in the specific case of Burgers transitions. Moreover, the demonstration is based on the decomposition of the rotational group of $\mathbf{G}^{\beta}$ into generator elements, but restrict $\mathbf{G}^{\beta}$ to the subgroup $\mathbf{R}^{\beta}=\left\{\mathrm{g}_{n}^{\beta} \in \mathbf{G}^{\beta} / \operatorname{det}\left(\mathrm{g}_{n}^{\beta}\right)=+1\right\}$ is in general incomplete. Indeed, a parent phase that has no rotational symmetries can generate variants; for example, $\mathbf{G}^{\beta}=$ $\{E, \mathrm{~m}\}$ can generate two variants.

Remark 4. We have shown that $\mathbf{V}^{\alpha}$ can be considered as a group if and only if $\mathbf{H}^{\beta} \triangleleft \mathbf{G}^{\beta}$. Another special condition has been described by Van Tendeloo \& Amelinckx (1974): when $\exists \mathbf{K}^{\beta} \leq \mathbf{G}^{\beta}$ such that $\mathbf{G}^{\beta}=\mathbf{H}^{\beta} \mathbf{K}^{\beta}, \mathbf{H}^{\beta} \cap \mathbf{K}^{\beta}=\{E\}$ and $\left|\mathbf{H}^{\beta}\right|\left|\mathbf{K}^{\beta}\right|=$ $\left|\mathbf{G}^{\beta}\right|$. In this particular case, it is said that it is always possible to find in each left coset $\alpha_{i}$ one symmetry operation $\mathrm{g}_{i}^{\beta}$ (a coset representative) such that the set of these representatives $\left\{\mathrm{g}_{i}^{\beta}, i \in\left[1, N^{\alpha}\right]\right\}$ has a group structure. This set is called the variant generating group VGG. However, this group is not representative of the complete and complex structure of $\mathbf{V}^{\alpha}$. Indeed, the two examples in Figs. 1(b) and $(c)$ have the same $\mathrm{VGG}=\left\{E, r_{+\pi / 4}^{\beta}, r_{-\pi / 4}^{\beta}, I\right\}$, whereas these two cases are distinct; this means that the information about the OR of the variants with their parent crystal is lost if only the VGG is considered.

\subsection{Duality between the direct and inverse transitions}

This part is only relevant for structural phase transitions. In the previous section, we have introduced for the $\beta \rightarrow \alpha$ phase transition the intersection group (18): $\mathbf{H}^{\beta}=\mathbf{G}^{\beta} \cap \mathrm{T}_{>} \mathbf{G}^{\alpha} \mathrm{T}_{>}^{-1}$ with $\mathrm{T}_{>}=\left[\mathrm{B}_{1}^{\beta} \triangleright \mathrm{B}_{1}^{\alpha_{1}}\right]$. Let us now consider the inverse transition $\alpha \rightarrow \beta$ that can be obtained for example by increasing the temperature. The intersection group for this transition is $\mathbf{H}^{\alpha}=$ $\mathbf{G}^{\alpha} \cap \mathrm{T}_{<} \mathbf{G}^{\beta} \mathrm{T}_{<}^{-1} \leq \mathbf{G}^{\alpha}$ with $\mathrm{T}_{<}=\left[\mathrm{B}_{1}^{\alpha} \triangleright \mathrm{B}_{1}^{\beta_{1}}\right]$. One can choose $\alpha=$ $\alpha_{1}$ and $\beta_{1}=\beta$; it follows that

$$
\mathrm{T}_{<}=\mathrm{T}_{>}^{-1}
$$

and, therefore,

$$
\mathbf{H}^{\alpha}=\mathrm{T}_{<} \mathbf{H}^{\beta} \mathrm{T}_{<}^{-1}=\mathrm{T}_{<} \mathbf{H}^{\beta} \mathrm{T}_{>} .
$$

Thus, the two groups $\mathbf{H}^{\alpha}$ and $\mathbf{H}^{\beta}$ are linked by the isomorphism $\psi$ introduced in equation (17): $\mathbf{H}^{\beta} \cong_{\psi} \mathbf{H}^{\alpha}$. Both groups may be understood as the largest subgroups of $\mathbf{G}^{\alpha}$ and $\mathbf{G}^{\beta}$ linked by the isomorphism $\psi$. One may also notice that

$$
\begin{aligned}
\mathrm{T}_{<} \mathbf{H}^{\beta} & =\mathbf{H}^{\alpha} \mathrm{T}_{<} \\
\mathbf{H}^{\beta} \mathrm{T}_{>} & =\mathrm{T}_{>} \mathbf{H}^{\alpha} .
\end{aligned}
$$

Thus, $\left(\mathbf{H}^{\beta}, \mathbf{H}^{\alpha}\right)$ appears as the solutions of the equation with unknown $\left(g^{\beta}, g^{\alpha}\right) \in\left(\mathbf{G}^{\beta}, \mathbf{G}^{\alpha}\right): g^{\beta} \mathrm{T}_{>}=\mathrm{T}_{>} g^{\alpha}$, i.e. as the elements of $\mathbf{G}^{\beta} \mathrm{T}_{>} \cap \mathrm{T}_{>} \mathbf{G}^{\alpha}$, i.e. as the intersection of the trivial external $\mathbf{G}^{\beta}$ orbit with the trivial internal $\mathbf{G}^{\alpha}$ orbit on the set $\mathbf{G}^{\beta} \mathrm{T}_{>} \mathbf{G}^{\alpha}$ (see $\S \S 3.3$ and 4.2). 
Since $\mathbf{H}^{\beta} \cong{ }_{\psi} \mathbf{H}^{\alpha},\left|\mathbf{H}^{\beta}\right|=\left|\mathbf{H}^{\alpha}\right|$ and, since $\mathbf{N}^{\alpha}=\left|\mathbf{G}^{\beta}\right| /\left|\mathbf{H}^{\beta}\right|$ and $\mathrm{N}^{\beta}=\left|\mathbf{G}^{\alpha}\right| /\left|\mathbf{H}^{\alpha}\right|$, we deduce a simple equation that links the numbers of variants of the direct and inverse transitions:

$$
\mathbf{N}^{\alpha}\left|\mathbf{G}^{\alpha}\right|=\mathbf{N}^{\beta}\left|\mathbf{G}^{\beta}\right| \text {. }
$$

To illustrate this equation, we consider the two-dimensional case of Fig. 1: since $\left|\mathbf{G}^{\beta}\right|=8$ and $\left|\mathbf{G}^{\alpha}\right|=6$, the number of variants of the inverse transition is $\mathrm{N}^{\beta}=6$ in the case of Fig. $1(a)$ (because $\mathrm{N}^{\alpha}=8$ ), and $\mathrm{N}^{\beta}=3$ in the cases Figs. $1(b)$ and $(c)$ (because $\left.\mathrm{N}^{\alpha}=4\right)$.

From equation (33), one may also notice that, if $\left|\mathbf{G}^{\beta}\right|=\left|\mathbf{G}^{\alpha}\right|$, then $\left|\mathbf{N}^{\beta}\right|=\left|\mathbf{N}^{\alpha}\right|$. This means that the number of variants generated by a transition is equal to the number of variants generated by the inverse transition, whatever the orientation relationship. This is the case for the martensitic transition in steels $\gamma \rightarrow \alpha$ (see $\$ 9.2)$.

The $\beta \rightarrow \alpha$ Landau transition corresponds to $\mathbf{H}^{\beta}=\psi\left(\mathbf{G}^{\alpha}\right)$, which implies that $\left|\mathbf{H}^{\beta}\right|=\left|\mathbf{G}^{\alpha}\right|$. Equation (23) applied to the inverse $\alpha \rightarrow \beta$ transition leads to $\mathrm{N}^{\beta}=\left|\mathbf{G}^{\alpha}\right| /\left|\mathbf{H}^{\alpha}\right|=\left|\mathbf{G}^{\alpha}\right| /\left|\mathbf{H}^{\beta}\right|=1$. This means that all the crystals $\alpha_{i}$ (inherited from the same parent crystal $\beta_{1}$ ) generate only one variant of phase $\beta$ by the inverse transition (which is the initial parent crystal $\beta_{1}$ ). Thus, Landau transitions appear as reversible ones. As proved by (33), this is not the case for reconstructive transitions.

\section{Definition of the operators}

\subsection{Transformations from one variant to another}

Equation (19), $\mathbf{T}^{\beta \rightarrow \alpha_{i}}=\mathrm{g}_{i}^{\beta} \mathbf{H}^{\beta} \mathrm{T}_{>}$, can also be written with the help of equations (32):

$$
\mathbf{T}^{\beta \rightarrow \alpha_{i}}=\mathrm{g}_{i}^{\beta} \mathrm{T}_{>} \mathbf{H}^{\alpha} .
$$

This set of matrices corresponds to the transformations from a basis $\mathrm{B}_{1}^{\beta}$ to bases $\mathrm{B}_{k}^{\alpha_{i}}$ such that: $\mathrm{g}_{i}^{\beta}=\left[\mathrm{B}_{1}^{\beta} \triangleright \mathrm{B}_{i}^{\beta}\right] \in \mathbf{G}^{\beta}, \mathrm{T}_{>}=$ $\left[\mathrm{B}_{i}^{\beta} \triangleright \mathrm{B}_{1}^{\alpha_{i}}\right]$ and $\left[\mathrm{B}_{1}^{\alpha_{i}} \triangleright \mathrm{B}_{k}^{\alpha_{i}}\right]=\mathrm{h}_{k}^{\alpha_{i}} \in \mathbf{H}^{\alpha}$. Hence, the set of transformation matrices from a variant $\alpha_{i}$ to a variant $\alpha_{j}$, denoted $\mathbf{T}^{\alpha_{i} \rightarrow \alpha_{j}}$, is given by the matrices of the form $\left[\mathrm{B}_{k}^{\alpha_{i}} \triangleright \mathrm{B}_{l}^{\alpha_{j}}\right]=$ $\left[\mathrm{B}_{1}^{\beta} \triangleright \mathrm{B}_{k}^{\alpha_{i}}\right]^{-1}\left[\mathrm{~B}_{1}^{\beta} \triangleright \mathrm{B}_{l}^{\alpha_{j}}\right]$, with $\left[\mathrm{B}_{1}^{\beta} \triangleright \mathrm{B}_{k}^{\alpha_{i}}\right] \in \mathbf{T}^{\beta \rightarrow \alpha_{i}}$ and $\left[\mathrm{B}_{1}^{\beta} \triangleright \mathrm{B}_{l}^{\alpha_{j}}\right] \in$ $\mathbf{T}^{\beta \rightarrow \alpha_{\mathrm{j}}}$. Therefore,

$$
\mathbf{T}^{\alpha_{i} \rightarrow \alpha_{j}}=\left(\mathbf{T}^{\beta \rightarrow \alpha_{i}}\right)^{-1} \mathbf{T}^{\beta \rightarrow \alpha_{\mathrm{j}}}=\mathrm{T}_{>}^{-1} \mathbf{H}^{\beta} \mathrm{g}_{i j}^{\beta} \mathbf{H}^{\beta} \mathrm{T}_{>} .
$$

In the following we note

$$
\alpha_{i j}=\mathbf{H}^{\beta} \mathrm{g}_{i j}^{\beta} \mathbf{H}^{\beta}=\alpha_{i}^{-1} \alpha_{j} .
$$

Since $\alpha_{i j}=\psi\left(\mathbf{T}^{\alpha_{i} \rightarrow \alpha_{j}}\right)$ with $\psi$ the isomorphism defined in equation (17), there is a one-to-one correspondence between the set $\mathbf{T}^{\alpha_{i} \rightarrow \alpha_{j}}$ and the set $\mathbf{H}^{\beta} \mathrm{g}_{i j}^{\beta} \mathbf{H}^{\beta}$. Moreover, a trivial property of $\alpha_{i j}$ is that $\alpha_{i j}^{-1}=\alpha_{j i}$, which means that the set of transformation matrices from $\alpha_{j}$ to $\alpha_{i}$ is the inverse of the set of transformation matrices from $\alpha_{i}$ to $\alpha_{j}$.

\subsection{The set of operators}

It can be easily checked from equation (36) that all the sets $\alpha_{i j}$ with $(i, j) \in\left[1, \mathrm{~N}^{\alpha}\right]^{2}$ are either disjoint or equal. Each distinct $\alpha_{i j}$ is called an operator and denoted $\mathrm{O}_{n}^{\alpha}$ (the term 'operator' will be justified later). Each operator is a class of equality on the set of the sets of transformation matrices $\left\{\alpha_{\mathrm{ij}}\right\}$. In other words, $\alpha_{i j}$ and $\alpha_{k l}$ are elements of the same class $\mathrm{O}_{n}^{\alpha}$ if and only if $\alpha_{i j}=\alpha_{k l}\left(=\mathrm{O}_{n}^{\alpha}\right)$. An operator can be geometrically imagined as a color attributed to the arrows (transformations) that link the variants. The index $n=0$ is applied for all $\alpha_{i i}$ with $i \in\left[1, \mathrm{~N}^{\alpha}\right]$. One may verify that:

$$
\mathrm{O}_{0}^{\alpha}=\mathbf{H}^{\beta} \text {. }
$$

Each operator that transforms $\alpha_{i}$ into $\alpha_{j}$ is equal to

$$
\mathrm{O}_{n}^{\alpha}=\alpha_{i j}=\mathbf{H}^{\beta} \mathrm{g}_{n}^{\beta} \mathbf{H}^{\beta}, \quad \text { with } \quad \mathrm{g}_{n}^{\beta}=\mathrm{g}_{i j}^{\beta} .
$$

This shows that each operator takes the algebraic form of a double coset $\mathbf{H}^{\beta} \mathrm{g}_{n}^{\beta} \mathbf{H}^{\beta}$. In consequence, the set of operators, which will be denoted $\mathbf{O}^{\alpha}$, can be deduced from the canonical decomposition of the group $\mathbf{G}^{\beta}$ into double cosets:

$$
\mathbf{G}^{\beta}=\mathbf{H}^{\beta} \mathrm{g}_{1}^{\beta} \mathbf{H}^{\beta} \cup \mathbf{H}^{\beta} \mathrm{g}_{2}^{\beta} \mathbf{H}^{\beta} \cup \ldots \cup \mathbf{H}^{\beta} \mathrm{g}_{\mathrm{N}}^{\beta} \mathbf{o}^{\alpha} \mathbf{H}^{\beta} .
$$

Each double coset (operator) can be treated as a unique mathematical entity. The set of operators is the double quotient set, algebraically denoted $\mathbf{H}^{\beta} \backslash \mathbf{G}^{\beta} / \mathbf{H}^{\beta}$ :

$$
\mathbf{O}^{\alpha}=\mathbf{H}^{\beta} \backslash \mathbf{G}^{\beta} / \mathbf{H}^{\beta}=\left\{\mathbf{H}^{\beta} \mathrm{g}_{1}^{\beta} \mathbf{H}^{\beta}, \mathbf{H}^{\beta} \mathrm{g}_{2}^{\beta} \mathbf{H}^{\beta}, \ldots, \mathbf{H}^{\beta} \mathrm{g}_{\mathrm{N}^{\alpha}}^{\beta} \mathbf{H}^{\beta}\right\} .
$$

$\mathrm{N}^{\mathbf{0}^{\alpha}}$ is the number of operators, it is equal to the order of this set:

$$
\mathbf{N}^{\mathbf{o}^{\alpha}}=\left|\mathbf{H}^{\beta} \backslash \mathbf{G}^{\beta} / \mathbf{H}^{\beta}\right| .
$$

Remark 5. The double coset decomposition was introduced for Landau transitions by Janovec (1972) with details in Janovec \& Dvorakova (1989). ${ }^{\mathbf{8}}$ To keep Janovec's vocabulary for domain pairs, we say that an operator is ambivalent if $\mathbf{T}^{\alpha_{i} \rightarrow \alpha_{j}}=$ $\mathbf{T}^{\alpha_{j} \rightarrow \alpha_{i}} \Leftrightarrow \alpha_{i j}=\alpha_{j i} \Leftrightarrow \alpha_{i j}^{-1}=\alpha_{i j} \Leftrightarrow \mathbf{H}^{\beta} \mathrm{g}_{n}^{\beta} \mathbf{H}^{\beta}=\mathbf{H}^{\beta}\left(\mathrm{g}_{n}^{\beta}\right)^{-1} \mathbf{H}^{\beta} \Leftrightarrow$ $\mathrm{O}_{n}^{\alpha}=\left(\mathrm{O}_{n}^{\alpha}\right)^{-1}$. For ambivalent operators, the set of transformations from the variant $\alpha_{i}$ to the variant $\alpha_{j}$ is equal to the set of transformations between the variant $\alpha_{i}$ and the variant $\alpha_{j}$. One can then write $\mathbf{T}^{\alpha_{i} \leftrightarrow \alpha_{j}}$ instead of $\mathbf{T}^{\alpha_{i} \rightarrow \alpha_{j}}$. If the operator is not ambivalent, i.e. if $\mathbf{T}^{\alpha_{i} \rightarrow \alpha_{j}} \neq \mathbf{T}^{\alpha_{\mathrm{j}} \rightarrow \alpha_{\mathrm{i}}} \Leftrightarrow \mathrm{O}_{n}^{\alpha} \neq\left(\mathrm{O}_{n}^{\alpha}\right)^{-1}$, the operator is called polar. However, an ambivalent equivalence class can be created from polar operators by writing $\mathbf{T}^{\alpha_{i} \leftrightarrow \alpha_{j}}=$ $\mathbf{T}^{\alpha_{i} \rightarrow \alpha_{j}} \cup \mathbf{T}^{\alpha_{j} \rightarrow \alpha_{i}}=\psi^{-1}\left(\left\langle\mathrm{O}_{n}^{\alpha}\right\rangle\right)$ with $\left\langle\mathrm{O}_{n}^{\alpha}\right\rangle=\mathrm{O}_{n}^{\alpha} \cup\left(\mathrm{O}_{n}^{\alpha}\right)^{-1}$.

To illustrate our reasoning, let us consider the example of Fig. 1(a). One may verify with equation (38) that the set of variants $\mathbf{V}^{\alpha}$ and the set of transformations that link the variants, $\mathbf{O}^{\alpha}$, are isomorphic by the trivial application $\mathrm{p}(\mathbf{x})=$ $\{\mathbf{x}\}: \mathbf{O}^{\alpha} \cong_{\mathrm{p}} \mathbf{V}^{\alpha}$. Thus the number of operators is equal to the number of variants and $\mathbf{O}^{\alpha}$ has the same group structure as $\mathbf{V}^{\alpha}$. In the case of Fig. $1(b)$, it can be checked algebraically that $\mathrm{O}_{0}^{\alpha}=\left\{E, m_{x}^{\beta}\right\}, \mathrm{O}_{1}^{\alpha}=\alpha_{12}=\alpha_{21}=\alpha_{23}=\alpha_{32}=\alpha_{34}=\alpha_{43}=\alpha_{41}=\alpha_{14}=$ $\left\{m_{x y}^{\beta}, r_{+\pi / 2}^{\beta}, r_{-\pi / 2}^{\beta}, m_{x \bar{y}}^{\beta}\right\}$ and $\mathrm{O}_{2}^{\alpha}=\alpha_{13}=\alpha_{31}=\alpha_{24}=\alpha_{42}=\left\{I, m_{y}^{\beta}\right\}$.

${ }^{8}$ Janovec \& Dvorakova (1989) use the double coset decomposition to define classes of ordered pair of states $\left(\mathbf{S}_{i}, \mathbf{S}_{j}\right)$. Two domain pairs $\left(\mathbf{S}_{i}, \mathbf{S}_{j}\right)$ and $\left(\mathbf{S}_{k}, \mathbf{S}_{l}\right)$ are called 'crystallographically equivalent' if $\exists g^{\beta} \in G^{\beta}$ such that $\mathbf{S}_{j}=g^{\beta} \mathbf{S}_{i}$ and $\mathbf{S}_{l}=g^{\beta} \mathbf{S}_{k}$. There is a one-to-one correspondence between the class of pair domains and the double coset. 
We may see that all the operators are ambivalent. We also point out that neither $\mathbf{V}^{\alpha}$ nor $\mathbf{O}^{\alpha}$ has a group structure.

Although the decomposition (39) is canonical (see footnote 8), Janovec \& Dvorakova (1989) did not give a general formula to calculate the number of double cosets. However, we will show now that such a generalization is possible. The reader is invited to look over Appendix $B$ before reading the next section.

\subsection{Numbering the operators}

We consider the operator $\mathrm{O}_{n}^{\alpha}=\mathbf{H}^{\beta} \mathrm{g}_{n}^{\beta} \mathbf{H}^{\beta}$. In this set, each right coset $\mathbf{H}^{\beta} \mathrm{g}_{n}^{\beta}$ can be written as a set of matrices $\left\{\mathrm{g}_{i}^{\beta}, \mathrm{g}_{i}^{\beta} \in\right.$ $\mathbf{H}^{\beta} \mathrm{g}_{n}^{\beta}$. Hence, any operator (double coset) can be written as a set of left cosets:

$$
\mathrm{O}_{n}^{\alpha}=\left\{\mathrm{g}_{i}^{\beta} \mathbf{H}^{\beta}, \mathrm{g}_{i}^{\beta} \in \mathbf{H}^{\beta} \mathrm{g}_{n}^{\beta}\right\}=\left\{\alpha_{i} \text { with } \mathrm{g}_{i}^{\beta} \in \mathbf{H}^{\beta} \mathrm{g}_{n}^{\beta}\right\} .
$$

Thus, according to the vocabulary of Janovec \& Dvorakova (1989), if each operator comprises one coset, it is called simple or, if it comprises many cosets, it is called multiple. The isotropic operator $\mathrm{O}_{0}^{\alpha}=\left\{\alpha_{1}\right\}$ is always a simple operator. In the example of Fig. 1(b), the three operators can be written $\mathrm{O}_{0}^{\alpha}=$ $\left\{\alpha_{1}\right\}, \mathrm{O}_{1}^{\alpha}=\left\{\alpha_{2}, \alpha_{4}\right\}$ and $\mathrm{O}_{2}^{\alpha}=\left\{\alpha_{3}\right\}$; only $\mathrm{O}_{1}^{\alpha}$ is multiple.

If $\mathbf{H}^{\beta}$ is normal in $\mathbf{G}^{\beta}, \mathbf{H}^{\beta} \mathrm{g}_{i}^{\beta}=\mathrm{g}_{i}^{\beta} \mathbf{H}^{\beta}$ (any right coset is equal to a left coset); consequently, any operator $\mathrm{O}_{n}^{\alpha}=\left\{\mathrm{g}_{i}^{\beta} \mathbf{H}^{\beta}\right\}=\left\{\alpha_{i}\right\}$. The trivial isomorphism $\mathrm{p}(\mathbf{x})=\{\mathbf{x}\}$ links each double coset to a left coset and, in consequence, $\mathbf{H}^{\beta} \backslash \mathbf{G}^{\beta} / \mathbf{H}^{\beta} \cong_{\mathrm{p}} \mathbf{G}^{\beta} / \mathbf{H}^{\beta}$ :

$$
\mathbf{H}^{\beta} \triangleleft \mathbf{G}^{\beta} \Rightarrow \mathbf{O}^{\alpha} \cong_{p} \mathbf{V}^{\alpha} \cong_{p} \mathbf{G}^{\beta} .
$$

Then, if $\mathbf{H}^{\beta} \triangleleft \mathbf{G}^{\beta}$, one can associate one variant to each operator (the operator is simple): the set of variants and the set of operators have the same group structure $\mathbf{G}^{\beta} / \mathbf{H}^{\beta}$. A consequence is $\mathbf{N}^{\mathbf{0}^{\alpha}}=\mathbf{N}^{\alpha}$. This is the case in Fig. 1(a).

More generally (whatever $\mathbf{H}^{\beta}$ is in $\mathbf{G}^{\beta}$ ), from equation (39) one can always notice that $\mathbf{H}^{\beta}$ acts at the left of the set of variants $\mathbf{V}^{\alpha}$. Then, each operator can be identified to a left $\mathbf{H}^{\beta}$ orbit on the set $\mathbf{V}^{\alpha}$. Since $\mathbf{H}^{\beta}$ acts closely on $\mathbf{V}^{\alpha}$, these orbits can be called internal orbits. To sum up, an operator is expressed by a double coset and represented by an internal $\mathbf{H}^{\beta}$ orbit on $\mathbf{V}^{\alpha}$. A direct consequence is that the number of operators $\mathbf{N}^{\boldsymbol{\alpha}^{\alpha}}$ is the number of $\mathbf{H}^{\beta}$ orbits on $\mathbf{V}^{\alpha}$; this number appears in the class equation (Appendix $B$ ):

$$
\left|\mathbf{V}^{\alpha}\right|=\sum_{n=0}^{\mathrm{N}^{\mathbf{0}^{\alpha}}-1}\left|\mathrm{O}_{n}^{\alpha}\right|=\sum_{n=1}^{\mathrm{N}^{\mathbf{0}^{\alpha}}} \frac{\left|\mathbf{H}^{\beta}\right|}{\left|\mathbf{S t a b}_{\mathbf{H}^{\beta}}\left(\alpha_{i}\right)\right|}, \quad \text { with } \quad \alpha_{i} \in \mathrm{O}_{\mathrm{n}}^{\alpha} .
$$

Since $\left|\mathbf{V}^{\alpha}\right|=\mathrm{N}^{\alpha}$ and $\mathrm{O}_{0}^{\alpha}=\left\{\alpha_{1}\right\}$, it follows that

$$
\mathrm{N}^{\alpha}=1+\sum_{n=1}^{\mathrm{N}^{\mathbf{0}^{\alpha}}-1} \mathrm{~N}_{\alpha_{i}}^{\mathrm{O}_{\mathrm{n}}^{\alpha}}, \quad \text { with } \quad \mathrm{N}_{\alpha_{i}}^{\mathrm{O}_{\mathrm{n}}^{\alpha}}=\frac{\left|\mathbf{H}^{\beta}\right|}{\left|\mathbf{S t a b}_{\mathbf{H}^{\beta}}\left(\alpha_{i}\right)\right|} \in \mathbb{N},
$$

where $\mathrm{N}_{\alpha_{i}}^{\mathrm{O}_{n}^{\alpha}}$ is the number of variants $\alpha_{i}$ contained in each orbit $\mathrm{O}_{n}^{\alpha}$ and $\mathbf{S t a b}_{\mathbf{H}^{\beta}}\left(\alpha_{i}\right)=\left\{h^{\beta} \in \mathbf{H}^{\beta}, h^{\beta} \alpha_{i}=\alpha_{i}\right\}$ is the stabilizer of $\alpha_{i}$ in $\mathbf{H}^{\beta}$.

Since $\operatorname{Stab}_{\mathbf{H}^{\beta}}\left(\alpha_{i}\right)=\mathbf{H}^{\beta} \cap \mathrm{g}_{i}^{\beta} \mathbf{H}^{\beta}\left(\mathrm{g}_{i}^{\beta}\right)^{-1}$ with $\mathrm{g}_{i}^{\beta} \in \alpha_{i}$ (see the end of Appendix $B$ ) and since $\mathrm{g}_{i}^{\beta} \mathbf{H}^{\beta}\left(\mathrm{g}_{i}^{\beta}\right)^{-1}=\mathbf{H}^{\beta / \alpha_{i}}$ [equation (26)], it follows that

$$
\operatorname{Stab}_{\mathbf{H}^{\beta}}\left(\alpha_{i}\right)=\mathbf{H}^{\beta} \cap \mathbf{H}^{\beta / \alpha_{i}} .
$$

From this formula, $\mathbf{S t a b}_{\mathbf{H}^{\beta}}\left(\alpha_{i}\right)$ appears as the set of elements that leaves invariant both $\alpha_{1}$ and $\alpha_{i}$, i.e. that leaves invariant the ordered pair $\left(\alpha_{1}, \alpha_{i}\right)$.

Equation (44) proves that the number of operators is always lower than the number of variants, and that the number of variants is the addition of the unity to some terms that divide $\left|\mathbf{H}^{\beta}\right|$. To illustrate this property, we consider the case of Fig. $1(b)$ : there are $\mathrm{N}^{\alpha}=4$ variants that are partitioned into three operators $\left\{\alpha_{1}\right\},\left\{\alpha_{2}, \alpha_{4}\right\}$ and $\left\{\alpha_{3}\right\}$ whose number of elements divides $\left|\mathbf{H}^{\beta}\right|=2$ such that finally $4=1+2+1$. Since there are three terms in this decomposition, $\mathrm{N}^{\mathbf{0}^{\alpha}}=3$. The reader can also check in this example that equation (45) gives the stabilizer of the three operators $\left\{\alpha_{1}\right\},\left\{\alpha_{2}, \alpha_{4}\right\}$ and $\left\{\alpha_{3}\right\}$.

The number of operators $\mathrm{N}^{\mathbf{0}^{\alpha}}$ given by equation (44) can be also directly obtained from the Burnside formula (see Appendix B):

$$
\mathbf{N}^{\mathbf{0}^{\alpha}}=\frac{1}{\left|\mathbf{H}^{\beta}\right|} \sum_{h^{\beta} \in \mathbf{H}^{\beta}}\left|\mathbf{F i x}_{\mathbf{V}^{\alpha}}\left(h^{\beta}\right)\right|,
$$

where $\mathbf{F i x}_{\mathbf{V}^{\alpha}}\left(h^{\beta}\right)=\left\{\alpha_{i} \in \mathbf{V}^{\alpha}, h^{\beta} \alpha_{i}=\alpha_{i}\right\}$ is the fixer of $h^{\beta}$ in $\mathbf{V}^{\alpha}$.

In equation (42), $\left\{\alpha_{i}\right\}=\left\{\mathrm{g}_{i}^{\beta} \mathbf{H}^{\beta}\right.$ with $\left.\mathrm{g}_{i}^{\beta} \in \mathbf{H}^{\beta} \mathrm{g}_{n}^{\beta}\right\}$ is also equal to $\left\{\mathbf{H}^{\beta} \alpha_{i}, \mathrm{~g}_{i}^{\beta} \in \mathbf{H}^{\beta} \mathrm{g}_{n}^{\beta}\right\}=\left\{\alpha_{1}^{-1} \alpha_{i}\right.$ with $\left.\mathrm{g}_{i}^{\beta} \in \mathbf{H}^{\beta} \mathrm{g}_{n}^{\beta}\right\}$; consequently,

$$
\mathrm{O}_{n}^{\alpha}=\left\{\alpha_{i}\right\}=\left\{\alpha_{1 i}\right\}
$$

This means that the variants $\alpha_{i}$ that constitute the operator $\mathrm{O}_{n}^{\alpha}$ in equation (42) are all the 'images' of the variant $\alpha_{1}$ by the operator $\mathrm{O}_{n}^{\alpha}$. Equation (47) can also be written

$$
\alpha_{1} \mathrm{O}_{n}^{\alpha}=\left\{\alpha_{i}\right\},
$$

which should be read from left to right: 'first take $\alpha_{1}$, then apply $\mathrm{O}_{n}^{\alpha}$ and you obtain different variants $\alpha_{i}^{\prime}$. With this equation, $\mathrm{O}_{n}^{\alpha}$ appears as an operator. Moreover, $\mathrm{O}_{n}^{\alpha}$ acts as a multivalued operation that transforms $\alpha_{1}$ into many $\alpha_{i}$. The pairs $\left(\alpha_{i}, \alpha_{j}\right)$ verifying $\alpha_{i j}=\mathrm{O}_{n}^{\alpha}$ are the pairs (source, target) for the operator $\mathrm{O}_{n}^{\alpha}(\operatorname{see} \S 7.1)$.

Remark 6. In this work, the information required to build the different variants inherited from the same parent crystal is obtained from the set of equivalent transformations defined in (19): $\mathbf{T}^{\beta \rightarrow \alpha_{i}}=\mathrm{g}_{i}^{\beta} \mathrm{T}_{>} \mathbf{H}^{\alpha}$. However, the whole set of transformations from the basis $\mathrm{B}_{1}^{\beta}$ to all the bases $\mathrm{B}_{n}^{\alpha_{i}}$ of any crystal $\alpha_{i}$ (not necessarily considered as a variant) is given by $\mathbf{T}_{t}^{\beta \rightarrow \alpha_{i}}=$ $\mathrm{g}_{i}^{\beta} \mathrm{T}_{>} \mathbf{G}^{\alpha}$, with the index $t$ for total. The difference between the two sets $\mathbf{T}_{t}^{\beta \rightarrow \alpha_{i}}$ and $\mathbf{T}^{\beta \rightarrow \alpha_{i}}$ forms excessive information that is not useful to determine the algebraic structure constituted by the variants. The total set of transformation matrices from a crystal $\alpha_{i}$ to a crystal $\alpha_{j}$ is $\mathbf{T}_{t}^{\alpha_{i} \rightarrow \alpha_{j}}=\mathbf{G}^{\alpha} \mathrm{T}_{>}^{-1} \mathrm{~g}_{i j}^{\beta} \mathrm{T}_{>} \mathbf{G}^{\alpha}$, which includes the set $\mathbf{T}^{\alpha_{i} \rightarrow \alpha_{j}}$ presented in equation (35). This is actually the set used in all the metallurgical studies devoted to the reconstruction of parent grains from EBSD maps, for example on martensitic steels (Gourgues et al., 2000) or on titanium alloys (Gey \& Humbert, 2003). Indeed, if $\mathbf{G}^{\alpha}$ is not reduced to $\{E\}$ or $\{E, I\}$, each set $\mathbf{T}_{t}^{\alpha_{i} \rightarrow \alpha_{j}}$ is a set of isometry matrices that contains at least one matrix with positive 
determinant, i.e. a rotational matrix. For each rotational matrix of the set $\mathbf{T}_{t}^{\alpha_{i} \rightarrow \alpha_{j}}$, the rotation angle and axis $(\delta, \mathrm{U})$ can be easily deduced from its eigenvalues and eigenvectors: $\cos (\delta)$ is known from the trace of the matrix and the sign of $\delta$ can be attributed if the reference coordinate system $\mathrm{B}_{1}^{\beta}$ is oriented as a direct basis. Then, at each set $\mathbf{T}_{t}^{\alpha_{i} \rightarrow \alpha_{j}}$, one may associate a representative pair $\left(\delta_{\min }, \mathrm{U}\right)$ with the minimum angle and the corresponding rotation axis in order to create a class of transformations: two transformations $\mathbf{T}_{t}^{\alpha_{i} \rightarrow \alpha_{j}}$ and $\mathbf{T}_{t}^{\alpha_{k} \rightarrow \alpha_{l}}$ are declared 'equal' or 'distinct' by comparing their corresponding pairs $\left(\delta_{\min }, \mathrm{U}\right)_{i j}$ and $\left(\delta_{\min }, \mathrm{U}\right)_{k l}$. Actually, in the previous metallurgical studies, only $|\delta|$ has been taken into consideration [probably because only $\cos (\delta)$ was considered in the rotational matrices]. The operators presented in these studies (they are called 'special misorientations') are therefore less precise since they are all forced to be ambivalent: they correspond to the operators of type $\left\langle\mathrm{O}_{n}^{\alpha}\right\rangle$. We have determined by computation of the operators $\left\langle\mathrm{O}_{n}^{\alpha}\right\rangle$ and the corresponding pairs $\left(\left|\delta_{\min }\right|, \mathrm{U}\right)$ from the operators $\mathrm{O}_{n}^{\alpha}$ defined by equation (47) for any phase transition; the results for Burgers transitions are presented in $\$ 9.1$

\section{Groupoid of orientational variants}

We have seen that in general $\mathbf{V}^{\alpha}$, the set of variants, does not have a group structure. What could be the algebraic structure of such a set? Moreover, what is the algebraic structure of $\mathbf{O}^{\alpha}$, the set of operators that link these variants? Since all the terms have been mathematically defined in the previous sections, it will be quite direct to show that the combination of two sets $\mathbf{V}^{\alpha}$ and $\mathbf{O}^{\alpha}$ forms a groupoid structure. These considerations will allow us to show that the composition of operators is a multivalued operation and how it is possible to establish a composition table for the operators. This section must be considered as a theoretical approach; an example of the application of the groupoids to materials-science problems will be given in $\$ 10.1$ with more details in Cayron et al. (2006).

\subsection{Introduction to groupoids}

A group defines a structure of actions without explicitly presenting the objects on which these actions are applied. Indeed, the actions of the group $\mathbf{G}$ applied to the identity element $e$ implicitly define the objects of the set $\mathbf{G}$ by $g e=g$; in other terms, in a group, actions and objects are two isomorphic entities. A groupoid enlarges the notion of group by explicitly introducing, in addition to the actions, the objects on which the actions are applied. By this approach, many identities may exist (they correspond to the actions that leave an object invariant). We present here a definition adapted from the Weinstein (1996) paper about the transformation groupoids: let $x$ be a solid and $\boldsymbol{\Gamma}$ a finite subset of the group of isometries $\mathbf{O}(3)$, and let us call $\mathbf{X}$ the set of similar solids formed by the actions of $\boldsymbol{\Gamma}$ on $x$. The transformation groupoid formed by $\mathbf{X}$ and $\Gamma$ is the set:
$\mathbf{G}(\mathbf{X}, \boldsymbol{\Gamma})=\left\{g=(x, \gamma, y)\right.$ with $(x, y) \in \mathbf{X}_{2}, \gamma \in \boldsymbol{\Gamma}$ and $\left.x \gamma=y\right\}$

with the partially defined operations: $(x, \gamma, y)(y, v, z)=$ $(x, \gamma \nu, z)$. One may imagine $\gamma$ of $\boldsymbol{\Gamma}$ as an arrow (without reference to any object of $\mathbf{X})$ and $g$ of $\mathbf{G}(\mathbf{X}, \boldsymbol{\Gamma})$ as the arrow $\gamma$ from the object $x$ to the object $y$. Contrary to the convention used by Weinstein, the composition of the binary operations $\gamma \nu$ must be understood as 'first apply $\gamma$ and then apply $\nu$ '. This convention is the direct consequence of the definition of transformation matrices presented in $\$ 3.1$ and of their composition rule (6).

We denote by $s$ and $t$ two maps (called source and target) from $\mathbf{G}(\mathbf{X}, \boldsymbol{\Gamma})$ onto $\mathbf{X}$ such that:

$$
s:(x, \gamma, y) \rightarrow x \text { and } t:(x, \gamma, y) \rightarrow y
$$

The operations on $\mathbf{G}(\mathbf{X}, \boldsymbol{\Gamma})$ have the following properties.

1 . The product is defined only for certain pairs of elements of $\mathbf{G}: g h$ is defined only when $t(g)=s(h)$.

2. It is associative: if either the product $(g h) k$ or $g(h k)$ is defined, then so is the other and they are equal.

3 . For each $g \in \mathbf{G}$, there are left and right identity elements $\lambda_{g}$ and $\rho_{g}$ such that $\lambda_{g} g=g=g \rho_{g}$.

4. Each $g$ of $\mathbf{G}$ has an inverse $g^{-1}$ for which $g g^{-1}=\lambda_{g}$ and $g^{-1} g=\rho_{g}$.

More generally, a groupoid with a base $\mathbf{X}$ is a set $\mathbf{G}(\mathbf{X}, \Gamma)$ with mappings $s$ and $t$ from $\mathbf{G}(\mathbf{X}, \boldsymbol{\Gamma})$ onto $\mathbf{X}$ and a partially defined binary operation $(g, h) \Rightarrow g h$ satisfying the four previous conditions. One may consider each $g$ of $\mathbf{G}$ as the arrow $s(g) \Rightarrow t(g)$. This direction is more natural than the one resulting from the Weinstein convention. ${ }^{9}$ Some concepts are also important in groupoid theory:

(a) The isotropy group of $x \in \mathbf{X}$ consists of those $g$ in $\mathbf{G}(\mathbf{X}, \boldsymbol{\Gamma})$ with $s(g)=x=t(g)$.

(b) An orbit of the groupoid $\mathbf{G}(\mathbf{X}, \boldsymbol{\Gamma})$ containing $x$ is $\mathbf{O}_{x}=$ $\{g=(x, \gamma, y), x \gamma=y$ and $\gamma \in \Gamma\}$. The set of orbits over $\mathbf{X}$ defines an equivalence class with $x \sim_{\mathbf{G}} y$ if and only if there is a groupoid element $g$ such that $s(g)=x$ and $t(g)=y$; i.e. $x \gamma=y$.

(c) We need to introduce a new notion, the 'operator', to explain the results obtained in the present study. Let us consider $\left(x_{1}, y_{1}\right)$ and $\left(x_{2}, y_{2}\right) \in \mathbf{X}^{2}$ such that $x_{1} \gamma=y_{1}$ and $x_{2} v=$ $y_{2}$. We introduce the equivalence relation on the pairs by: $\left(x_{1}, y_{1}\right) \sim\left(x_{2}, y_{2}\right) \Leftrightarrow \gamma=v$. It is the relation of pairs of elements $(x, y)$ similarly placed in the groupoid. The equivalence classes partition the groupoid $\mathbf{G}$ according to the sets of elements $g=$ $(x, \gamma, y)$ such that $x$ and $y$ are linked by the same binary operation. The binary operation associated with each set of this partition is what we call an operator.

In order to illustrate the definition of groupoid, let us consider a simple one-dimensional example with the set of the relative integers associated with the operation + (addition), but omitting, for instance, the numbers 5 and 11:

\footnotetext{
${ }^{9}$ Weinstein's convention leads to the opposite: $t(g) \rightarrow s(g)$ which appears confusing. This linguistic ambiguity comes from the fact that Weinstein has chosen the convention of the functional composition $f \circ g$ ('first apply $g$ and then $f$ '). In this paper, we have preferred the notation $f g$ ('first apply $f$ and then $g$ ') the composition of the linear functions $f$ and $g$ associated with transformation matrices $\mathrm{F}$ and $\mathrm{G}$ (footnote 2 ).
} 
$\mathrm{S}=\mathbb{Z}-\{5,11\}$. Of course, $(\mathbb{Z},+)$ is a group, but not $(\mathrm{S},+)$. However, one can associate with $\mathrm{S}$ the structure $\mathbf{G}(\mathrm{S},+)$, defined by the set of elements $g=\left(\mathrm{z}_{1}, \mathrm{z}_{2}-\mathrm{z}_{1}, \mathrm{z}_{2}\right)$ with $\left(\mathrm{z}_{1}, \mathrm{z}_{2}\right) \in$ $S^{2}$ and the composition rule $\left(z_{1}, z_{2}-z_{1}, z_{2}\right)\left(z_{2}, z_{3}-z_{2}, z_{3}\right)=$ $\left(\mathrm{z}_{1}, \mathrm{z}_{3}-\mathrm{z}_{1}, \mathrm{z}_{3}\right)$. One may check that $\mathbf{G}(\mathrm{S},+)$ is a groupoid. This kind of groupoid is called a pair or banal groupoid. The identities are $g_{0}=\left(\mathrm{z}_{1}, 0, \mathrm{z}_{1}\right)$ and, if $g=\left(\mathrm{z}_{1}, \mathrm{z}_{2}-\mathrm{z}_{1}, \mathrm{z}_{2}\right), g^{-1}=$ $\left(z_{2}, z_{1}-z_{2}, z_{1}\right)$. For any fixed number $Z$, the equivalence class of elements $\left(z_{1}, z_{2}-z_{1}, z_{2}\right)$ linked by the same operation $z_{2}-z_{1}=+Z$ forms the operator $+Z$. This must be understood by: $\mathrm{z}_{1}+\mathrm{Z}=\mathrm{z}_{2}$ ('first consider $\mathrm{z}_{1}$, then apply $+\mathrm{Z}$, and you obtain $\left.\mathrm{z}_{2}^{\prime}\right)$.

\subsection{Definition and properties of the groupoid of orienta- tional variants}

In the previous section, we have defined the set of variants $\mathbf{V}^{\alpha}=\left\{\alpha_{i}\right\}=\mathbf{G}^{\beta} / \mathbf{H}^{\beta}$ for the $\beta \rightarrow \alpha$ transition. The transformation from the variant $\alpha_{i}$ to the variant $\alpha_{j}$ was defined by $\mathbf{T}^{\alpha_{i} \rightarrow \alpha_{j}}$ $=\mathrm{T}_{>}^{-1} \alpha_{i j} \mathrm{~T}_{>}$, with $\alpha_{i j}=\alpha_{i}^{-1} \alpha_{j}$ being an element of the set $\mathbf{O}^{\alpha}=$ $\mathbf{H}^{\beta} \backslash \mathbf{G}^{\beta} / \mathbf{H}^{\beta}$. Now, it may be checked that $\mathbf{V}^{\alpha}$, the set of variants, associated with $\mathbf{O}^{\alpha}$, the set of operators, forms a groupoid that we call a groupoid of orientational variants:

$$
\begin{aligned}
\mathbf{G}^{\beta \rightarrow \alpha} & =\mathbf{G}\left(\mathbf{V}^{\alpha}, \mathbf{O}^{\alpha}\right) \\
& =\left\{\left(\alpha_{i}, \alpha_{i j}, \alpha_{j}\right), \alpha_{i} \in \mathbf{V}^{\alpha}, \alpha_{i j} \in \mathbf{O}^{\alpha}, \alpha_{j} \in \mathbf{V}^{\alpha}, \alpha_{i} \alpha_{i j}=\alpha_{j}\right\}
\end{aligned}
$$

with the partially binary operation $\left(\alpha_{i}, \alpha_{i j}, \alpha_{j}\right)\left(\alpha_{j}, \alpha_{j k}, \alpha_{k}\right)=$ $\left(\alpha_{i}, \alpha_{i j} \alpha_{j k}, \alpha_{k}\right)=\left(\alpha_{i}, \alpha_{i k}, \alpha_{k}\right)$, as illustrated in Fig. 2. In all the following, we will directly associate $\alpha_{i j}$ with $\left(\alpha_{i}, \alpha_{i j}, \alpha_{j}\right)$. The variants $\alpha_{i}$ and $\alpha_{j}$ appear as the source and target of $\alpha_{i j}$ : $\alpha_{i}=$ $s\left(\alpha_{i j}\right)$ and $\alpha_{j}=t\left(\alpha_{i j}\right)$. One may imagine $\alpha_{i j}$ as an arrow pointing from $\alpha_{i}$ to $\alpha_{j}$.

The groupoid $\mathbf{G}^{\beta \rightarrow \alpha}$ is geometrically rather simple, i.e. its low-level properties can be visualized or verified from the algebraic definition (50).

1. The binary operation is associative: the products $\left(\alpha_{i j} \alpha_{j k}\right) \alpha_{k l}$ and $\alpha_{i j}\left(\alpha_{j k} \alpha_{k l}\right)$ are equal (to $\left.\alpha_{i l}\right)$.

2. For each $\alpha_{i j}$ in $\mathbf{G}^{\beta \rightarrow \alpha}$, there are left and right identity elements that are both equal to $\alpha_{i i}=\alpha_{j j}=\mathbf{H}^{\alpha}=\mathrm{O}_{0}^{\alpha}$, which also constitutes the isotropy group of each variant $\alpha_{i}$.

3. Each element $\alpha_{i j}$ in $\mathbf{G}^{\beta \rightarrow \alpha}$ has an inverse equal to $\alpha_{i j}^{-1}=$ $\alpha_{j i}$.

In the groupoid $\mathbf{G}^{\beta \rightarrow \alpha}$, all the binary operations between the variants are defined; consequently, there is only one trivial orbit on the base $\mathbf{V}^{\alpha}$. However, there are many operators in $\mathbf{G}^{\beta \rightarrow \alpha}$ and their set is $\mathbf{O}^{\alpha}$. For each operator $\mathrm{O}_{n}^{\alpha}$ of $\mathbf{O}^{\alpha}$, one can associate a set of pairs $\left\{\left(\alpha_{i}, \alpha_{j}\right),\left(\alpha_{k}, \alpha_{l}\right), \ldots\right\}$ of variants similarly oriented in the parent crystal, i.e. such that $\alpha_{i j}=\alpha_{k l}=$ $\ldots=\mathrm{O}_{n}^{\alpha}$.

This definition of groupoid is very general. Any polycrystal (a set of randomly oriented crystals) can actually be imagined as a groupoid. If there are $N$ crystals in this polycrystal, there will be, in general, $2 \mathrm{C}(N, 2)$ operators that link these crystals. What makes the groupoid of orientational variants interesting relies on the fact that the variants are not randomly oriented and, consequently, the number of operators is far lower than
$2 \mathrm{C}(N, 2)$. Moreover, these operators can be composed in a special way and this composition may be seen as the groupoid signature, as shown in the following.

\subsection{Composition of operators}

We can perform two interesting compositions between the operators of the groupoid of orientational variants.

The first one is: $\left(\mathrm{O}_{m}^{\alpha}, \mathrm{O}_{n}^{\alpha}\right) \in\left(\mathbf{O}^{\alpha}\right)^{2} \rightarrow \mathrm{O}_{m}^{\alpha} \mathrm{O}_{n}^{\alpha}=\left\{\mathrm{O}_{q}^{\alpha}\right\} \in \mathrm{P}\left(\mathbf{O}^{\alpha}\right)$, where $\mathrm{P}\left(\mathbf{O}^{\alpha}\right)$ is the set of partitions of the set $\mathbf{O}^{\alpha}$. This composition can be easily computed from the associativity condition of the groupoid. Indeed, we write $\mathrm{O}_{m}^{\alpha}$ as a set of $\alpha_{i j}$ and $\mathrm{O}_{n}^{\alpha}$ as a set of $\alpha_{k l}$. Then, we find in each set the elements that verify $t\left(\alpha_{i j}\right)=\mathrm{s}\left(\alpha_{k l}\right)$, i.e. $j=k$, and write $\alpha_{i l}=\alpha_{i j} \alpha_{k l}$. Since each $\alpha_{i l}$ belongs to a class $\mathrm{O}_{q}^{\alpha}$, the product of operators $\mathrm{O}_{m}^{\alpha} \mathrm{O}_{n}^{\alpha}$ takes the form of a set $\left\{\mathrm{O}_{q}^{\alpha}\right\}$.

The second one is: $\left(\mathrm{O}_{m}^{\alpha}, \mathrm{O}_{n}^{\alpha}\right) \in\left(\mathbf{O}^{\alpha}\right)^{2} \rightarrow\left(\mathrm{O}_{m}^{\alpha}\right)^{-1} \mathrm{O}_{n}^{\alpha}=\left\{\mathrm{O}_{q}^{\alpha}\right\} \in$ $\mathrm{P}\left(\mathbf{O}^{\alpha}\right)$. This composition can also be easily computed by taking $\alpha_{1}$ as a reference. Indeed, we write $\mathrm{O}_{m}^{\alpha}$ as a set of $\alpha_{i}\left(=\alpha_{1 i}\right)$ and $\mathrm{O}_{n}^{\alpha}$ as a set of $\alpha_{j}\left(=\alpha_{1 j}\right)$. Then, we directly write $\alpha_{i j}=\alpha_{1 i}^{-1} \alpha_{1 j}$. In general, the composition of two operators is neither reduced to $\emptyset$ nor to a singleton. It means that the composition is not a classical application but a multivalued product. The multivalued composition of operators is geometrically visible. In the example of Fig. 1(b), since $\mathrm{O}_{0}^{\alpha}=\left\{E, m_{x}^{\beta}\right\}, \mathrm{O}_{1}^{\alpha}=\alpha_{12}=\alpha_{21}=$ $\alpha_{23}=\alpha_{32}=\alpha_{34}=\alpha_{43}=\alpha_{41}=\alpha_{14}$ and $\mathrm{O}_{2}^{\alpha}=\alpha_{13}=\alpha_{31}=\alpha_{24}=\alpha_{42}$, it may be checked that $\left(\mathrm{O}_{1}^{\alpha}\right)^{-1} \mathrm{O}_{2}^{\alpha}=\left\{\mathrm{O}_{1}^{\alpha}\right\},\left(\mathrm{O}_{1}^{\alpha}\right)^{-1} \mathrm{O}_{1}^{\alpha}=\left\{\mathrm{O}_{0}^{\alpha}, \mathrm{O}_{2}^{\alpha}\right\}$ and $\left(\mathrm{O}_{2}^{\alpha}\right)^{-1} \mathrm{O}_{2}^{\alpha}=\left\{\mathrm{O}_{0}^{\alpha}\right\}$. In this simple case, the multivalued result obtained with $\left(\mathrm{O}_{1}^{\alpha}\right)^{-1} \mathrm{O}_{1}^{\alpha}$ comes from the ambivalence of $\mathrm{O}_{1}^{\alpha}$; but this is not always the reason (as will be shown for a more complex case given in §9.1). The composition $\left(\mathrm{O}_{m}^{\alpha}, \mathrm{O}_{n}^{\alpha}\right) \rightarrow\left(\mathrm{O}_{m}^{\alpha}\right)^{-1} \mathrm{O}_{n}^{\alpha}$ has the advantage of allowing the representation of the entire structure of the groupoid (variants and operators) in the same table that we will call table of composition of the groupoid (see Fig. 6 for example, with details in §9.1). This table plays the same role as a group table; it is the signature of the groupoid of orientational variants and is characteristic of the crystallographic aspects of the phase transition.

\subsection{Comparison to space groupoids}

Groupoids were first introduced in crystallography for order-disorder (OD) structures by Dornberger-Schiff \& Grell-Niemann (1961); after that the same authors noticed the partial character of some symmetry operations in twin structures (Dornberger-Schiff, 1959). OD structures are crystallographic structures constituted by two-dimensional periodic layers linked by partial (or local) operations (PO). The name

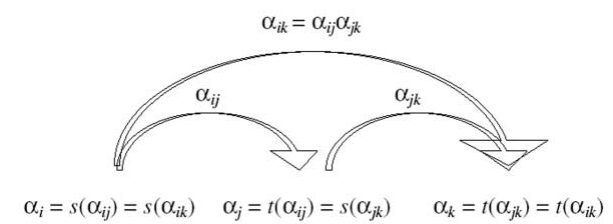

Figure 2

Composition of the operators in the groupoid of orientational variants $\mathrm{G}^{\beta \rightarrow \alpha}$. 
'order-disorder' is probably too broad since only the order in the stacking of the layers is not complete (the positions of the atoms inside each layer are entirely determined). The OD theory is actually a crystallographic (and algebraic) description of polytypism, but order-disorder transitions are far more universal and complex than order-disorder in polytypes. Dornberger-Schiff points out that the set of POs does not form a group but a groupoid since, for example, the operation that transforms the layer $L_{p}$ in $L_{q}$ and the operation that transforms the layer $L_{r}$ in $L_{s}$ cannot be combined, unless $q=r$. Such considerations explain some diffraction enhancements of symmetry not due to Friedel's law ${ }^{\mathbf{1 0}}$ in polytypes (Sadanaga, 1978; Sadanaga \& Ohsumi, 1979) and in quasicrystals (Yamamoto \& Ishihara, 1988). Those works introduce the concept of space groupoid in order to define the local symmetry operations on a space group, assuming that this one is constituted by a repeated substructure. Thus, the space groupoid and the groupoid of orientational variants could appear very different since the related problems are different. However, both can be considered as the algebraic description of a composite structure built from specially placed subunits and therefore are similar from a geometrical point of view. Their algebraic definitions are actually very close. ${ }^{11}$

\section{Calculation of the possible parent crystals}

\subsection{Motivations for the calculation}

We denote by $\theta_{c}$ the transition temperature for Landau transitions, and by $\theta_{\mathrm{s}}$ and $\theta_{\mathrm{f}}$ the start and finish temperatures for reconstructive transitions. We call 'half a cycle' the operation that consists in cooling the material from a temperature $\theta_{1}>\theta_{\mathrm{s}}\left(\right.$ or $\left.\theta_{\mathrm{c}}\right)$ to a temperature $\theta_{2}<\theta_{\mathrm{f}}\left(\right.$ or $\left.\theta_{\mathrm{c}}\right)$ or in heating it from $\theta_{2}$ to $\theta_{1}$. By 'transition cycle' we mean the succession of the two operations: cooling from $\theta_{1}$ to $\theta_{2}$ and heating from $\theta_{2}$ to $\theta_{1}$. Now, let us consider a transition cycle: we start at $\theta_{1}$ with a material constituted only by one crystal $\beta_{1}$; this material is then cooled to $\theta_{2}$ and completely transformed by the transition $\beta_{1} \rightarrow\left\{\alpha_{i}\right\}$. The material is now constituted by $\mathrm{N}^{\alpha}$ orientational crystals $\left\{\alpha_{i}\right\}$. It is then reheated at $\theta_{1}$ such that each crystal $\alpha_{i}$ becomes a parent crystal for the transformation $\alpha_{i} \rightarrow\left\{\beta_{k}\right\}$ (end of a cycle). We call these new crystals of phase $\beta$, which are variants of variants, $\beta$ crystals of second generation. Their number is denoted as $\mathrm{N}_{2}^{\beta}$. Since many of them are identical (in the same orientation), one may verify that

\footnotetext{
$\overline{\mathbf{1 0}}$ This approach generalizes Friedel's law, but still respects Curie's principle (Curie, 1894): the effects (diffraction) are at least as symmetric as the causes (the crystallographic structure).

${ }^{11}$ In his approach, Sadanaga uses the groupoid of the form $M=\left\{h_{i} \mathbf{H} h_{j}^{-1},\left(h_{i}, h_{i}\right) \in \mathbf{T}\right\}$, where $\mathbf{H}$ is the space group of the substructure (what they called the kernel of the groupoid, i.e. what we call the isotropy group of the substructure) and $\mathbf{T}$ is the hull of the groupoid (the set of actions of the groupoid, i.e. the operators). In Sadanaga's approach, the composite structure must be built from only one substructure, as is the case in the groupoid of orientational variants. We think that the difference between his groupoid $M$ and the groupoid of orientational variants $\left\{\mathbf{H} h_{i}^{-1} h_{j} \mathbf{H},\left(h_{i}, h_{j}\right) \in \mathbf{G}\right\}$ with $\mathbf{G}$ the point group of the parent crystal and $\mathbf{H}$ a subgroup of $\mathbf{G}$ is due to the difference in the composition rules.
}

$$
\mathrm{N}_{2}^{\beta} \leq \mathrm{N}^{\alpha} \mathrm{N}^{\beta}
$$

For Landau transitions, since $\mathrm{N}^{\beta}=1$, all the variants $\alpha_{i}$ recreate the same orientational crystal, which is $\beta_{1}$; and, in consequence, during thermal cycles, $\mathrm{N}_{2}^{\beta}=\mathrm{N}_{3}^{\beta}=\ldots=\mathrm{N}_{\infty}^{\beta}=\mathrm{N}^{\beta}=$ 1. For reconstructive transitions, to our knowledge, there is no general formula that gives $\mathrm{N}_{2}^{\beta}$.

This theoretical 'thermal cycling problem' is close to the one encountered in parent grain reconstruction from EBSD data. Only the orientations of a limited number of daughter grains is known in the data but one would like to reconstruct the parent grains and know their location and orientation. This is useful to determine if cracks occur and propagate at parent grain boundaries (Gourgues et al., 2000), or to better understand why and how variant selection occurs during successive heating and cooling treatments (Gey \& Humbert, 2003; Standford \& Bate, 2005). In order to determine the possible parent crystals of a finite set of variants $\left\{\alpha_{i}\right\}$, one may imagine the inverse transition such that each $\alpha_{i}$ becomes a parent crystal of a set of crystals $\left\{\beta_{k}\right\}$. The number of possible parent crystals of the crystals $\alpha_{i}$ is then given by the set of all the daughter crystals $\beta_{k}$ that have $\alpha_{i}$ as parent crystals. This problem has been treated by Humbert et al. (1995) for Burgers transitions. This section generalizes this approach with the help of the algebraic notions presented in the previous sections. Both problems (calculation of $\mathrm{N}_{2}^{\beta}$ and determination of parent grains) require the resolution of the same intermediate problem: assuming that the orientations of two variants are known, how many distinct crystals (i.e. with distinct orientations) can be their parent?

\subsection{Parent crystals common to two daughter crystals}

Let us consider two $\alpha$ variants inherited from a crystal $\beta_{1}$, for example $\alpha_{i}=\mathrm{g}_{i}^{\beta_{1}} \mathbf{H}^{\beta}$ and $\alpha_{j}=\mathrm{g}_{j}^{\beta_{1}} \mathbf{H}^{\beta} \in\left(\mathbf{V}^{\alpha}\right)^{2}$. These two

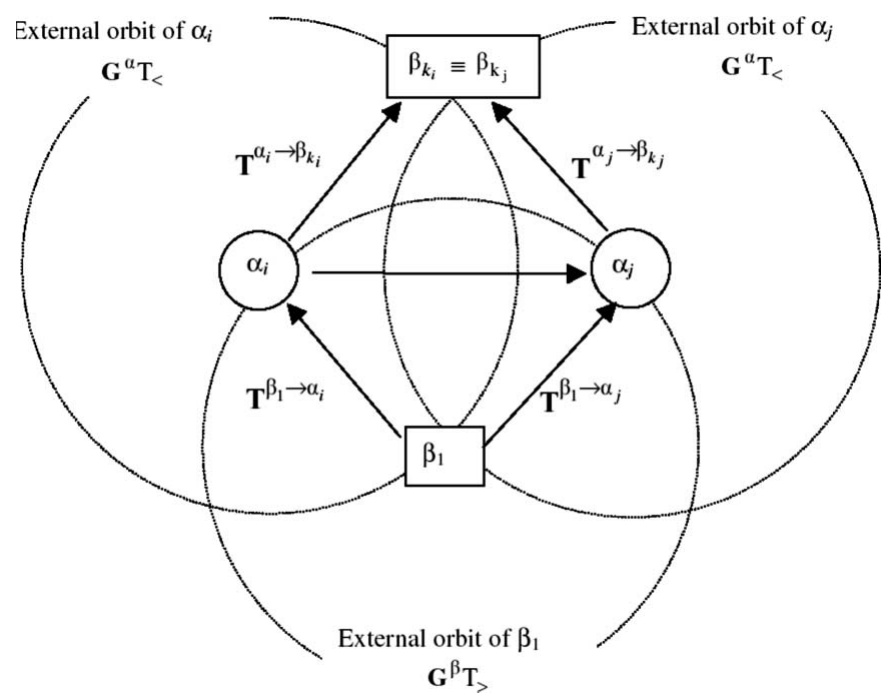

Figure 3

Intersection between the external $\mathbf{G}^{\alpha}$ orbit around $\alpha_{i}$ and the one around $\alpha_{j}$ : it allows one to know if two variants $\alpha_{i}$ and $\alpha_{j}$ inherited from a parent crystal $\beta_{1}$ can also be inherited from another parent crystal $\beta_{k}$. 
variants are determined from their respective orientations with the crystal $\beta_{1}$ given by equation (34):

$$
\begin{aligned}
& \mathbf{T}^{\beta_{1} \rightarrow \alpha_{i}}=\mathrm{g}_{i}^{\beta_{1}} \mathbf{H}^{\beta} \mathrm{T}_{>}=\alpha_{i} \mathrm{~T}_{>} \\
& \mathbf{T}^{\beta_{1} \rightarrow \alpha_{j}}=\mathrm{g}_{j}^{\beta_{1}} \mathbf{H}^{\beta} \mathrm{T}_{>}=\alpha_{j} \mathrm{~T}_{>} .
\end{aligned}
$$

We want to know if these two variants can also be inherited from another parent crystal, which we will call, for example, $\beta_{k}$. It is essential to notice that the index $k$ of this crystal is given with reference to the other crystals $\alpha_{i}$ or $\alpha_{j}$. Hence, if $\beta_{k}$ is inherited from the parent crystal $\alpha_{i}$ by the inverse transition, it must be denoted $\beta_{k_{i}}$ and, if it is inherited from the parent crystal $\alpha_{j}$, it must be denoted $\beta_{k_{j}}$ (see Fig. 3). Of course, $\beta_{k_{i}}$ and $\beta_{k_{i}}$ are both elements of the same set $\mathbf{V}^{\beta}$ but in general $k_{i} \neq k_{j}$. What is the algebraic relation between $\beta_{k_{i}}$ and $\beta_{k_{j}}$ ?

By inverting each member of the indexing convention chosen (12), we obtain

$$
\left[\mathrm{B}_{1}^{\alpha_{i}} \triangleright \mathrm{B}_{i}^{\beta_{1}}\right]=\left[\mathrm{B}_{1}^{\alpha_{1}} \triangleright \mathrm{B}_{1}^{\beta_{1}}\right]=\mathrm{T}_{<} .
$$

Then, by applying again the indexing rule (12), we can generalize:

$$
\left[\mathrm{B}_{k}^{\alpha_{i}} \triangleright \mathrm{B}_{i}^{\beta_{k}}\right]=\mathrm{T}_{<} .
$$

It follows that $\left[\mathrm{B}_{1}^{\alpha_{i}} \triangleright \mathrm{B}_{i}^{\beta_{k}}\right]=\left[\mathrm{B}_{1}^{\alpha_{i}} \triangleright \mathrm{B}_{k}^{\alpha_{i}}\right]\left[\mathrm{B}_{k}^{\alpha_{i}} \triangleright \mathrm{B}_{i}^{\beta_{k}}\right]=\mathrm{g}_{k}^{\alpha_{i}} \mathrm{~T}_{<}$. It must be noticed that $\mathrm{g}_{k}^{\alpha_{i}}$ is an element of $\mathbf{G}^{\alpha}$. By indicating its index in this list as $k_{i}$, we may write $\mathrm{g}_{k}^{\alpha_{i}}=\mathrm{g}_{k_{i}}^{\alpha}$. The crystal $\beta_{k}$ is then determined from two transformation matrices:

and

$$
\left[\mathrm{B}_{1}^{\alpha_{i}} \triangleright \mathrm{B}_{i}^{\beta_{k}}\right]=\mathrm{g}_{k_{i}}^{\alpha} \mathrm{T}_{<}
$$

$$
\left[\mathrm{B}_{1}^{\alpha_{j}} \triangleright \mathrm{B}_{j}^{\beta_{k}}\right]=\mathrm{g}_{k_{j}}^{\alpha} \mathrm{T}_{<} .
$$

These two transformation matrices are respectively elements of

and

$$
\mathbf{T}^{\alpha_{i} \rightarrow \beta_{k}}=\mathrm{g}_{k_{i}}^{\alpha} \mathbf{H}^{\alpha} \mathrm{T}_{<}=\beta_{k_{i}} \mathrm{~T}_{<}
$$

$$
\mathbf{T}^{\alpha_{j} \rightarrow \beta_{k}}=\mathrm{g}_{k_{j}}^{\alpha} \mathbf{H}^{\alpha} \mathrm{T}_{<}=\beta_{k_{j}} \mathrm{~T}_{<},
$$

with $\beta_{k_{i}}$ and $\beta_{k_{j}}$ elements of $\mathbf{V}^{\beta}$. One may notice that the matrices of $\mathbf{T}^{\alpha_{i} \rightarrow \beta_{k}}$ and $\mathbf{T}^{\alpha_{j} \rightarrow \beta_{k}}$ are elements of the external

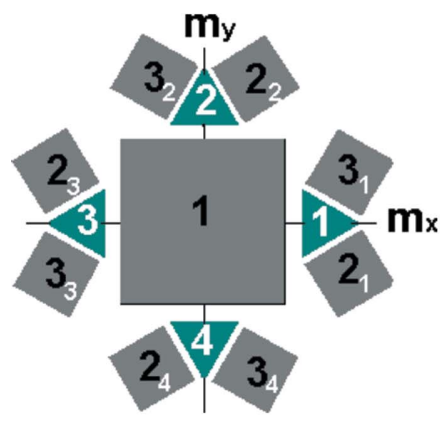

(a)

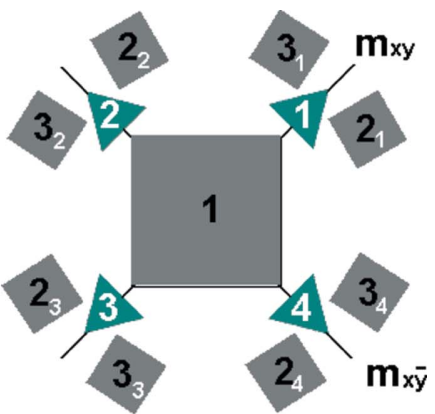

(b)
Figure 4

Variants of second generation $\beta_{k_{i}}$ corresponding (a) to Fig. $1(b)$ and $(b)$ to Fig. 1(c). They are generated from the variants $\alpha_{i}$ by the inverse transition $\alpha \rightarrow \beta$. Here, the number of distinct variants of second generation is $\mathrm{N}_{2}^{\beta}=3$. orbit $\mathbf{G}^{\alpha} \mathrm{T}_{<}$around $\alpha_{i}$ and $\alpha_{j}$, respectively (see Fig. 3). From equations (56), $\beta_{k}$ can be calculated in the same reference coordinate system $\mathrm{B}_{1}^{\beta_{1}}$ in two ways:

$$
\begin{aligned}
{\left[\mathrm{B}_{1}^{\beta_{1}} \triangleright \mathrm{B}_{i}^{\beta_{k}}\right] } & =\left[\mathrm{B}_{1}^{\beta_{1}} \triangleright \mathrm{B}_{1}^{\alpha_{i}}\right]\left[\mathrm{B}_{1}^{\alpha_{i}} \triangleright \mathrm{B}_{i}^{\beta_{k}}\right]=\mathrm{g}_{i}^{\beta} \mathrm{T}_{>} \mathrm{g}_{k_{i}}^{\alpha} \mathrm{T}_{<} \\
{\left[\mathrm{B}_{1}^{\beta_{1}} \triangleright \mathrm{B}_{j}^{\beta_{k}}\right] } & =\left[\mathrm{B}_{1}^{\beta_{1}} \triangleright \mathrm{B}_{1}^{\alpha_{i}}\right]\left[\mathrm{B}_{1}^{\alpha_{i}} \triangleright \mathrm{B}_{j}^{\beta_{k}}\right]=\mathrm{g}_{j}^{\beta_{3}} \mathrm{~T}_{>} \mathrm{g}_{k_{j}}^{\alpha} \mathrm{T}_{<} .
\end{aligned}
$$

These two transformation matrices define the same crystal $\beta_{k}$ if and only if, from equation (9),

$$
\begin{gathered}
{\left[\mathrm{B}_{1}^{\beta_{1}} \triangleright \mathrm{B}_{i}^{\beta_{k}}\right]^{-1}\left[\mathrm{~B}_{1}^{\beta_{1}} \triangleright \mathrm{B}_{j}^{\beta_{k}}\right] \in \mathbf{G}^{\beta} \Longleftrightarrow} \\
\mathrm{T}_{>}\left(\mathrm{g}_{k_{i}}^{\alpha}\right)^{-1} \mathrm{~T}_{<}\left(\mathrm{g}_{i}^{\beta}\right)^{-1} \mathrm{~g}_{j}^{\beta} \mathrm{T}_{>} \mathrm{g}_{k_{j}}^{\alpha} \mathrm{T}_{<} \in \mathbf{G}_{\beta} .
\end{gathered}
$$

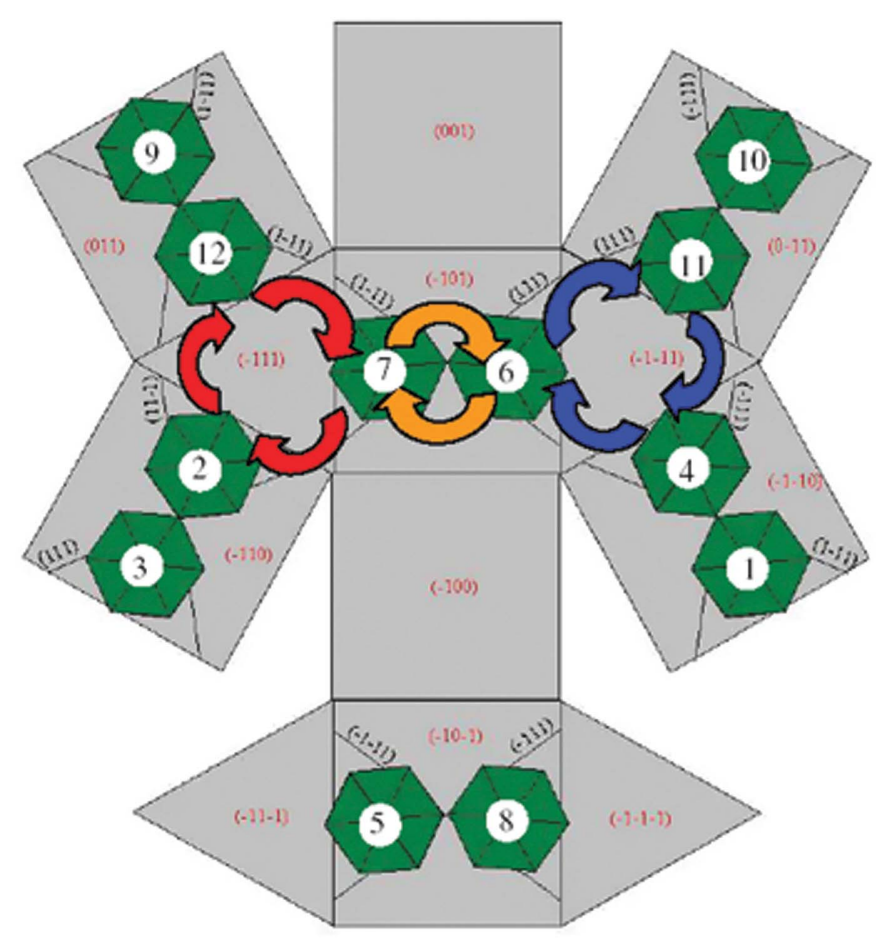

(a)

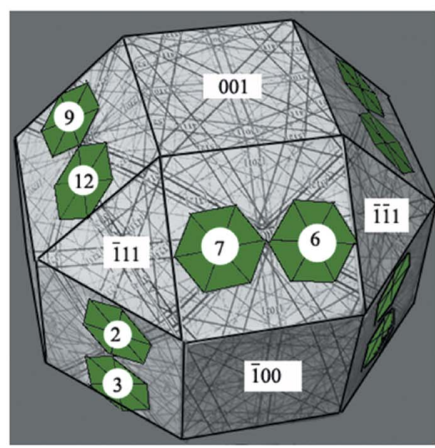

(b)

Figure 5

Representation of the $\alpha$ variants in $(a)$ a developed figure and $(b)$ a threedimensional figure. Some operators (see $\$ 7.2)$ are represented in $(a)$ : $\mathrm{O}_{2}^{\alpha}$, $\mathrm{O}_{3}^{\alpha}, \mathrm{O}_{5}^{\alpha}$ are in orange, red and blue, respectively. In $(b)$, the $\{100\},\{110\}$ and $\{111\}$ faces of the cubic $\beta$ parent crystal are in white, and the 12 hexagonal $\alpha$ variants are in green (only their $\{001\}$ planes are represented). 
By using equation (32), one may verify that equation (58) does not depend on the choice of representative $\mathrm{g}_{k_{i}}^{\alpha}$ and $\mathrm{g}_{k_{j}}^{\alpha}$ in their respective cosets $\mathrm{g}_{k_{i}}^{\alpha} \mathbf{H}^{\alpha}$ and $\mathrm{g}_{k_{i}}^{\alpha} \mathbf{H}^{\alpha}$ (both elements of $\mathbf{V}^{\beta}$ ). Consequently, only one representative can be chosen in each coset of $\mathbf{V}^{\beta}$ when using equation (58). This simplification is not possible for the choice of $\mathrm{g}_{i}^{\beta}$ and $\mathrm{g}_{j}^{\beta}$, however, since these two matrices are elements of $\alpha_{i}$ and $\alpha_{j}$, respectively, the matrix $\left(\mathrm{g}_{i}^{\beta}\right)^{-1} \mathrm{~g}_{j}^{\beta}=\mathrm{g}_{i j}^{\beta} \in \mathrm{O}_{n}^{\alpha}$, where $\mathrm{O}_{n}^{\alpha}$ is the operator that links $\alpha_{i}$ to $\alpha_{j}$. Testing all the $\mathrm{g}_{i j}^{\beta} \in \mathrm{O}_{n}^{\alpha}$ generates all the possible solutions of equation (58). Therefore, we conclude that two crystals $\alpha_{i}$ and $\alpha_{j}$ inherited from the same parent crystal $\beta_{1}$ and linked by $\mathrm{O}_{n}^{\alpha}$ can also be inherited from another crystal $\beta_{k}$ if and only if

$$
\exists\left(\beta_{k_{i}}, \beta_{k_{j}}\right) \in\left(\mathbf{V}^{\beta}\right)^{2}, \quad \mathrm{~T}_{>} \beta_{k_{i}}^{-1} \mathrm{~T}_{<} \mathrm{g}_{i j}^{\beta} \mathrm{T}_{>} \beta_{k_{j}} \mathrm{~T}_{<} \in \mathbf{G}^{\beta}
$$

with $\mathrm{g}_{i j}^{\beta} \in \mathrm{O}_{n}^{\alpha}$. One may check, with the help of equation (32), that $\beta_{1}=\mathbf{H}^{\alpha}$ always obeys equation (59). We recall that $k_{i}$ and $k_{j}$ represent the indices of the crystal $\beta_{k}$ with reference to the crystals $\alpha_{i}$ and $\alpha_{j}$, respectively. One can also verify that the operator $\mathrm{O}_{m}^{\beta}$ that links $\beta_{1}$ to $\beta_{k}$ is given by $\mathrm{O}_{m}^{\beta}=\beta_{1 k_{i}}=\beta_{1 k_{j}}=$ $\mathbf{H}^{\alpha} \beta_{k_{i}}=\mathbf{H}^{\beta} \beta_{k_{j}}$.

\subsection{Number of variants of second generation}

The variants of second generation corresponding to the cases of Figs. 1(b) and $(c)$ are presented in Figs. 4(a) and (b), respectively; their number can be geometrically determined; it is $\mathrm{N}_{2}^{\beta}=3$. In the present study, we do not propose a general algebraic formula that gives this number. We propose, however, a method that allows its calculation and that can be easily computed. We first consider the crystal $\alpha_{1}$ (inherited from $\left.\beta_{1}\right)$ and all its variants $\left\{\beta_{k_{1}}\right\}$. Then, we consider the second crystal $\alpha_{2}$ and all its variants $\left\{\beta_{k_{2}}\right\}$. By applying equation (59), we deduce all the variants of $\left\{\beta_{k_{2}}\right\}$ identical (internally equivalent) to one of the variants of $\left\{\beta_{k_{1}}\right\}$, and then the variants of $\left\{\beta_{k_{2}}\right\}$ that are 'really new' (those different from any $\left\{\beta_{k_{1}}\right\}$. Now, we consider the third variant $\alpha_{3}$ and all its variants $\left\{\beta_{k_{3}}\right\}$, and keep in this set only the new crystals (i.e. different from $\beta_{k_{1}}$ and $\beta_{k_{2}}$ ) etc. This algorithm has been applied to calculate the number of variants of second generation for complex cases such as those presented in $§ 9.1$. Of course, the mathematical study of the series of variant numbers $\mathrm{N}_{2}^{\beta}, \mathrm{N}_{3}^{\beta}, \ldots, \mathrm{N}_{n}^{\beta}$ of the $n$th generation would require a deeper study.

\section{Computation and application to reconstructive transitions}

The two-dimensional cases like those presented in Fig. 1 are in general geometrically simple. However, three-dimensional crystallographic cases encountered in materials science (phase transition, precipitation, twinning) are more complex. Therefore, we have written a computer program in Python language (Marteli, 2004), according to the equations presented in the previous sections, which calculates the set of variants, the set of operators, the composition table of the groupoid and the possible parent crystals inherited from two daughter variants. It takes a few seconds to compute all the variants and
Table 2

Intersection groups for a Burgers transition (OR in Table 1).

The symmetry operations are written with the Jones and with the Bradley \& Cracknel representations. See also Bradley \& Cracknell (1972) for details.

\begin{tabular}{lll}
\hline $\mathbf{H}^{\beta} \leq \mathbf{G}^{\beta}$ & $\psi$ & $\mathbf{H}^{\alpha} \leq \mathbf{G}^{\alpha}$ \\
\hline$E$ & $\rightarrow$ & $E$ \\
$I$ & $\rightarrow$ & $I$ \\
$(\bar{y} \bar{x} z)=\sigma_{\mathrm{da}}$ & $\rightarrow$ & $(x y \bar{z})=\sigma_{\mathrm{h}}$ \\
$(\mathrm{y} \mathrm{x} \bar{z})=\mathrm{C}_{2 a}$ & $\rightarrow$ & $(\bar{x} \bar{y} z)=\mathrm{C}_{2}$ \\
\hline
\end{tabular}

Table 3

For a Burgers transition, composition table between variants generating the operators: $\left(\alpha_{i}, \alpha_{j}\right) \rightarrow \alpha_{i j}=\alpha_{i}^{-1} \alpha_{j}=\mathrm{O}_{n}^{\alpha}$, where $\mathrm{O}_{n}^{\alpha}$ is the operator that links the variant $\alpha_{i}$ to the variant $\alpha_{j}$.

In this table, only the index $n$ of $\mathrm{O}_{n}^{\alpha}$ is reported $\left(\alpha_{i}\right.$ and $\alpha_{j}$ are in the first line and first column, respectively).

\begin{tabular}{lllllllllllll}
\hline & $\alpha_{1}$ & $\alpha_{2}$ & $\alpha_{3}$ & $\alpha_{4}$ & $\alpha_{5}$ & $\alpha_{6}$ & $\alpha_{7}$ & $\alpha_{8}$ & $\alpha_{9}$ & $\alpha_{10}$ & $\alpha_{11}$ & $\alpha_{12}$ \\
\hline$\alpha_{1}$ & 0 & 1 & 1 & 2 & 3 & 4 & 5 & 6 & 5 & 3 & 6 & 4 \\
$\alpha_{2}$ & 1 & 0 & 2 & 1 & 5 & 6 & 3 & 4 & 4 & 6 & 3 & 5 \\
$\alpha_{3}$ & 1 & 2 & 0 & 1 & 6 & 5 & 4 & 3 & 3 & 5 & 4 & 6 \\
$\alpha_{4}$ & 2 & 1 & 1 & 0 & 4 & 3 & 6 & 5 & 6 & 4 & 5 & 3 \\
$\alpha_{5}$ & 5 & 3 & 6 & 4 & 0 & 1 & 1 & 2 & 3 & 4 & 5 & 6 \\
$\alpha_{6}$ & 4 & 6 & 3 & 5 & 1 & 0 & 2 & 1 & 5 & 6 & 3 & 4 \\
$\alpha_{7}$ & 3 & 5 & 4 & 6 & 1 & 2 & 0 & 1 & 6 & 5 & 4 & 3 \\
$\alpha_{8}$ & 6 & 4 & 5 & 3 & 2 & 1 & 1 & 0 & 4 & 3 & 6 & 5 \\
$\alpha_{9}$ & 3 & 4 & 5 & 6 & 5 & 3 & 6 & 4 & 0 & 1 & 1 & 2 \\
$\alpha_{10}$ & 5 & 6 & 3 & 4 & 4 & 6 & 3 & 5 & 1 & 0 & 2 & 1 \\
$\alpha_{11}$ & 6 & 5 & 4 & 3 & 3 & 5 & 4 & 6 & 1 & 2 & 0 & 1 \\
$\alpha_{12}$ & 4 & 3 & 6 & 5 & 6 & 4 & 5 & 3 & 2 & 1 & 1 & 0 \\
\hline
\end{tabular}

Note that this table is not symmetric owing to the polarity of some operators. Indeed, operators 3 and 5 are distinguished: they correspond to a rotation of $-\pi / 3$ and $+\pi / 3$ around the $\langle 111\rangle$ directions of the cubic parent crystal oriented from the inside to the outside of the parent cube. They are represented in red and blue in Fig. 5, respectively.

operators with a $1 \mathrm{GHz} \mathrm{PC}$ for any crystalline structural transition. Such speed could not have been obtained without the theoretical considerations described in this paper. We give here the details of the results obtained for Burgers transitions; some results obtained for martensitic transitions in steels are also briefly reported.

\subsection{Burgers transitions}

The $\beta \rightarrow \alpha$ Burgers transitions (Burgers, 1934) are transitions from a high-temperature body-centered cubic phase $(\beta)$ to a low-temperature hexagonal close-packed phase $(\alpha)$. This transition occurs for example in $\mathrm{Ti}$ or $\mathrm{Zr}$ alloys. One of the possible ORs that links an $\alpha$ crystal to a $\beta$ parent crystal is given in Table 1. The intersection group $\mathbf{H}^{\beta}$ is given in Table 2 and the computed variants $\alpha_{i}$ are presented in Fig. 5. We found that $\left|\mathbf{H}^{\beta}\right|=4$ and, since $\left|\mathbf{G}^{\beta}\right|=48$, the number of $\alpha$ variants is $\mathbf{N}^{\alpha}=12$. The operators associated with the pairs of variants $\left(\alpha_{i}, \alpha_{j}\right)$ are determined by calculating all the distinct sets of matrices $\alpha_{i j}=\alpha_{i}^{-1} \alpha_{j}$, as presented in Table 3. We find that $\mathrm{O}_{0}^{\alpha}=$ $\left\{\alpha_{1}\right\}, \mathrm{O}_{1}^{\alpha}=\left\{\alpha_{2}, \alpha_{3}\right\}, \mathrm{O}_{2}^{\alpha}=\left\{\alpha_{4}\right\}, \mathrm{O}_{3}^{\alpha}=\left\{\alpha_{7}, \alpha_{9}\right\}, \mathrm{O}_{4}^{\alpha}=\left\{\alpha_{6}, \alpha_{12}\right\}$, $\mathrm{O}_{5}^{\alpha}=\left\{\alpha_{5}, \alpha_{10}\right\}$ and $\mathrm{O}_{6}^{\alpha}=\left\{\alpha_{8}, \alpha_{11}\right\}$. The number of operators is $\mathrm{N}^{\mathrm{O}^{\alpha}}=7$, in agreement with the class equation (44): $12=$ $1+2+1+2+2+2+2=$ addition of seven terms that each 
Table 4

For a Burgers transition, list of the operators $\mathrm{O}_{n}^{\alpha}$ and their corresponding variants (source and target) verifying $\mathrm{O}_{n}^{\alpha}=\alpha_{i j}$.

Each operator contains some rotations (see Remark 6); the minimum angle and some special angles $\left(60,90,120\right.$ or $\left.180^{\circ}\right)$ are indicated with their axis (in the source daughter basis).

\begin{tabular}{|c|c|c|}
\hline & $\begin{array}{l}\text { List of pair of variants }\left(\alpha_{i}, \alpha_{k}\right) \text {, source and } \\
\text { target of the operator } \mathrm{O}_{n}^{\alpha}\end{array}$ & $\begin{array}{l}\text { Rotation angle }\left({ }^{\circ}\right) \\
\text { and corresponding } \\
\text { axis }\end{array}$ \\
\hline $\mathrm{O}_{0}^{\alpha}$ & $\begin{array}{l}(1,1),(2,2),(3,3),(4,4),(5,5),(6,6),(7,7) \\
\quad(8,8),(9,9),(10,10),(11,11),(12,12)\end{array}$ & $\begin{array}{l}60^{\circ}[100] \\
120^{\circ}[100] \\
180^{\circ}[110]\end{array}$ \\
\hline $\mathrm{O}_{1}^{\alpha}$ & $\begin{array}{c}(1,2),(1,3),(2,1),(2,4),(3,1),(3,4),(4,2), \\
\quad(4,3),(5,6),(5,7),(6,5),(6,8),(7,5), \\
\quad(7,8),(8,6),(8,7),(9,10),(9,11),(10,9), \\
(10,12),(11,9),(11,12),(12,10),(12,11)\end{array}$ & $\begin{array}{l}90^{\circ}[1 \overline{90}] \\
180^{\circ}[63,36,35]\end{array}$ \\
\hline $\mathrm{O}_{2}^{\alpha}$ & $\begin{array}{c}(1,4),(2,3),(3,2),(4,1),(5,8),(6,7),(7,6), \\
(8,5),(9,12),(10,11),(11,10),(12,9)\end{array}$ & $\begin{array}{l}10.5^{\circ}[001] \\
180^{\circ}[1 \overline{9} 0]\end{array}$ \\
\hline $\mathrm{O}_{3}^{\alpha}$ & $\begin{array}{l}(1,7),(1,9),(2,5),(2,12),(3,6),(3,10) \\
\quad(4,8),(4,11),(5,1),(5,11),(6,4),(6,9) \\
\quad(7,2),(7,10),(8,3),(8,12),(9,3),(9,5) \\
\quad(10,1),(10,8),(11,2),(11,),(12,4) \\
\quad(12,7)\end{array}$ & $\begin{array}{l}60.8^{\circ}[1, \overline{9}, \overline{10}] \\
90^{\circ}[63,36,35] \\
120^{\circ}[\overline{7}, \overline{70}, 60]\end{array}$ \\
\hline $\mathrm{O}_{4}^{\alpha}$ & $\begin{array}{l}(1,6),(1,12),(2,8),(2,9),(3,7),(3,11) \\
\quad(4,5),(4,10),(5,4),(5,10),(6,1),(6,12), \\
\quad(7,3),(7,11),(8,2),(8,9),(9,2),(9,8) \\
\quad(10,4),(10,5),(11,3),(11,7),(12,1) \\
\quad(12,6)\end{array}$ & $\begin{array}{l}60^{\circ}[100] \\
180^{\circ}[8,16,5]\end{array}$ \\
\hline $\mathrm{O}_{5}^{\alpha}$ & $\begin{array}{l}(1,5),(1,10),(2,7),(2,11),(3,8),(3,9) \\
\quad(4,6),(4,12),(5,2),(5,9),(6,3),(6,11) \\
\quad(7,1),(7,12),(8,4),(8,10),(9,1),(9,6) \\
\quad(10,3),(10,7),(11,4),(11,5),(12,2) \\
\quad(12,8)\end{array}$ & 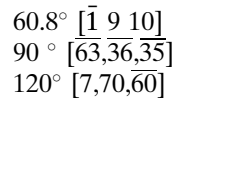 \\
\hline $\mathrm{O}_{6}^{\alpha}$ & $\begin{array}{l}(1,8),(1,11),(2,6),(2,10),(3,5),(3,12) \\
\quad(4,7),(4,9),(5,3),(5,12),(6,2),(6,10) \\
\quad(7,4),(7,9),(8,1),(8,11),(9,4),(9,7) \\
\quad(10,2),(10,6),(11,1),(11,8),(12,3) \\
(12,5)\end{array}$ & $\begin{array}{l}63.2^{\circ}[501] \\
120^{\circ}[\overline{450} 0] \\
180^{\circ}[\overline{1221}, 627,592]\end{array}$ \\
\hline
\end{tabular}

divides $\left|\mathbf{H}^{\beta}\right|=4$. For each operator, the list of source and target pairs $\left(\alpha_{i}, \alpha_{j}\right)$ is directly deduced from Table 3 and presented in Table 4 . We have also calculated the set of transformation matrices $\mathbf{T}_{t}^{\alpha_{i} \rightarrow \alpha_{j}}$ (see Remark 6) and we have reported some rotations with the minimum angle and with special angles (60, 90,120 or $180^{\circ}$ ) in the last column of Table 4 . One may notice that the operators $\mathrm{O}_{3}^{\alpha}$ and $\mathrm{O}_{5}^{\alpha}$ are complementary polar operators and that the other ones are ambivalent. If the operators are forced to be ambivalent (i.e. $\mathrm{O}_{3}^{\alpha}$ and $\mathrm{O}_{5}^{\alpha}$ considered as the same operator), we find that $\mathrm{N}^{\left\langle\mathrm{O}^{\alpha}\right\rangle}=6$, as published by Gey \& Humbert (2003). The composition of the operators is presented in Fig. 6. All the parent crystals of two variants $\alpha_{1}$ and $\alpha_{i},\left\{\beta_{1}, \ldots, \beta_{k}\right\}$ are calculated according to equation (59) and are given in Table 5. From this table, we recursively calculate if a set $\left\{\alpha_{1}, \ldots, \alpha_{N}\right\}$ of $N$ daughter variants can have only one parent crystal $\left(\beta_{1}\right)$. We found that four variants determine unambiguously the parent crystal. Indeed, in contrast to the results given by Humbert et al. (1995), in some special cases, even three variants $\left\{\alpha_{1}, \alpha_{6}, \alpha_{12}\right\}$ can be inherited from two different parent crystals $\beta_{1}$ and $\beta_{6}$, where $\beta_{6}$ is a twin of $\beta_{1}$ through the (111) mirror plane. The situation can be understood by observing on Fig. 5(a) that $\alpha_{1}$, $\alpha_{6}, \alpha_{12}$ have their basic vector $\mathbf{a}=[100]$ normal to the plane (111) of $\beta_{1}$ and, consequently, are invariant by the rotation of $180^{\circ}$ around $[100]_{\alpha_{1}}=[1 \overline{1} 1]_{\beta_{1}}$, which transforms $\beta_{1}$ into $\beta_{6}$. This solution has been forgotten by Humbert et al. (1995) probably because in that study only the rotational symmetries were taken into consideration. Moreover, from Table 5 and Table 4, we have determined that the number of variants of second generation is $\mathrm{N}_{2}^{\beta}=40$.

\subsection{Martensitic transitions in steels}

In the $\gamma \rightarrow \alpha$ martensitic transitions in steels, the parent phase is the face-centered cubic austenite $(\gamma)$ and the daughter phase, at low carbon levels, is the body-centered cubic martensite $\left(\alpha^{\prime}\right)$. There are two possible orientation relationships: the Nishiyama-Wasserman (NW) OR (Nishiyama, 1934; Wassermann, 1933) and the Kurdjumov-Sachs (KS) OR (Kurdjumov \& Sachs, 1930). Some results on the groupoid of variants are briefly reported here; more details will be given by Cayron et al. (2006). Since the parent and daughter phases have the same point-group order, the number of variants is independent of the direction of the transition arrow (heating or cooling). We find that $\left|\mathbf{H}^{\gamma}\right|=\left|\mathbf{H}^{\alpha}\right|=4$ and $\mathbf{N}^{\alpha}=\mathbf{N}^{\gamma}=12$ variants for a NW OR, and that $\left|\mathbf{H}^{\gamma}\right|=\left|\mathbf{H}^{\alpha}\right|=2$ and $\mathrm{N}^{\alpha}=\mathrm{N}^{\gamma}=24$ for a KS OR, which is well known in metallurgy. The operators are determined by computation, we find $\mathrm{N}^{\mathrm{O}^{\alpha}}=7$ with a NW OR and $\mathrm{N}^{\mathrm{O}^{\alpha}}=24$ with a KS OR. If the

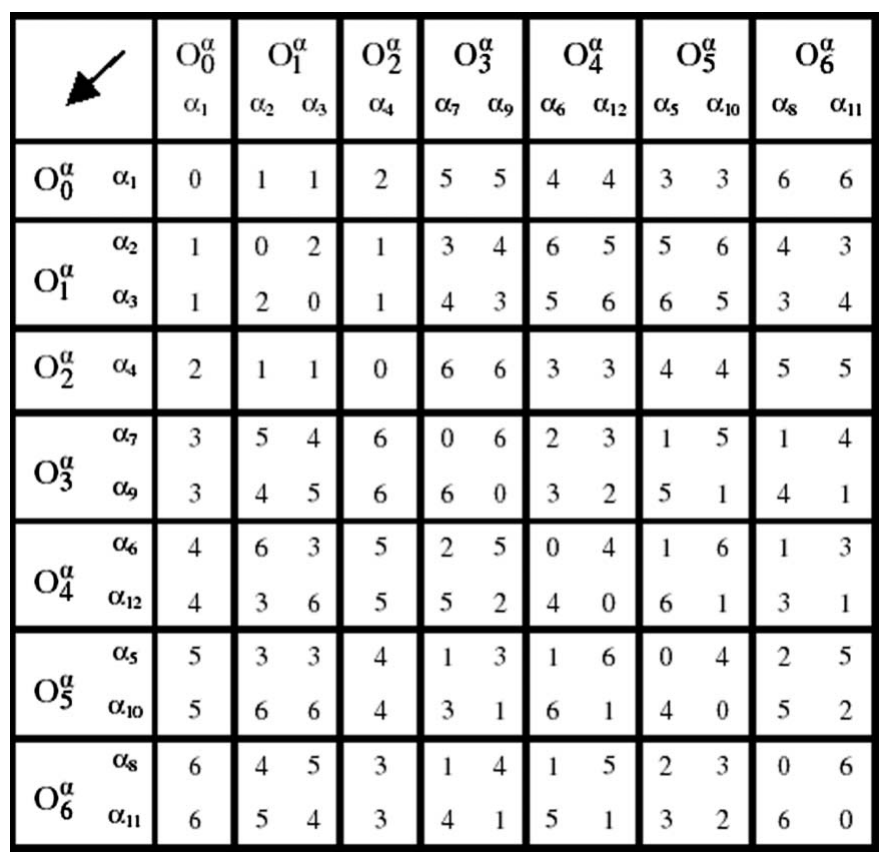

Figure 6

Composition table of the groupoid $\mathbf{G}^{\beta \rightarrow \alpha}$ for a Burgers transition. The table is deduced from Table 4 according to $\left(\mathrm{O}_{m}^{\alpha}, \mathrm{O}_{n}^{\alpha}\right) \rightarrow\left(\mathrm{O}_{m}^{\alpha}\right)^{-1} \mathrm{O}_{n}^{\alpha}=$ $\left\{\mathrm{O}_{q}^{\alpha}\right\}=\left\{\mathrm{O}_{q}^{\alpha}\right\}$ such that $\mathrm{O}_{q}^{\alpha}=\alpha_{i j}$ with $\alpha_{1 i} \in \mathrm{O}_{m}^{\alpha}$ and $\left.\alpha_{1 j} \in \mathrm{O}_{n}^{\alpha}\right\}$. Only the indices $q$ of $\mathrm{O}_{q}^{\alpha}$ are noted in the table $\left(\mathrm{O}_{m}^{\alpha}\right.$ and $\mathrm{O}_{n}^{\alpha}$ are in the first line and first column, respectively). For each operator $\mathrm{O}_{m}^{\alpha}$ and $\mathrm{O}_{n}^{\alpha}$, the corresponding variants $\alpha_{i}$ and $\alpha_{j}$ such that $\mathrm{O}_{m}^{\alpha}=\left\{\alpha_{1 i}\right\}$ and $\mathrm{O}_{n}^{\alpha}=\left\{\alpha_{1 j}\right\}$ are also reported. 
Table 5

For a Burgers transition $\beta \rightarrow \alpha$, list of the possible parent crystal $\left\{\beta_{j}\right\}$ of a pair of variants $\left(\alpha_{1}, \alpha_{i}\right)$.

\begin{tabular}{|c|c|c|c|c|c|c|c|c|c|c|c|c|}
\hline$\alpha_{1}$ & $\alpha_{1}$ & $\alpha_{2}$ & $\alpha_{3}$ & $\alpha_{4}$ & $\alpha_{5}$ & $\alpha_{6}$ & $\alpha_{7}$ & $\alpha_{8}$ & $\alpha_{9}$ & $\alpha_{10}$ & $\alpha_{11}$ & $\alpha_{12}$ \\
\hline$\left\{\beta_{j}\right\}$ & $\beta_{1} \ldots \beta_{6}$ & $\beta_{1}$ & $\beta_{1}$ & $\beta_{1} \beta_{3} \beta_{2}$ & $\beta_{1}$ & $\beta_{1} \beta_{6}$ & $\beta_{1}$ & $\beta_{1}$ & $\beta_{1}$ & $\beta_{1}$ & $\beta_{1}$ & $\beta_{1} \beta_{6}$ \\
\hline
\end{tabular}

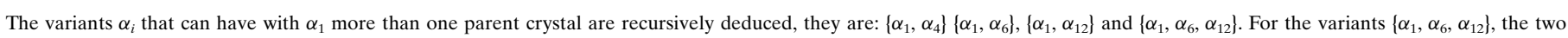
possible parent crystals are $\beta_{1}$ and $\beta_{6}$ : they are $\Sigma 3$ twins (see Fig. 5).

operators are forced to be ambivalent, we find that $\mathrm{N}^{\mathrm{O}^{\alpha}}=6$ with a NW OR and 17 with a KS OR, as published by Gourgues et al. (2000). All the parent crystals of two variants $\alpha_{1}$ and $\alpha_{i},\left\{\beta_{1}, \ldots, \beta_{k}\right\}$, are determined by computation; we have deduced that four and seven distinct variants determine unambiguously the parent crystal for a NW OR and a KS OR, respectively.

\section{Applications and perspectives}

This study generalizes the previous crystallographic studies on the Landau transitions to any type of crystalline transitions (Landau and reconstructive). We have introduced two equivalence classes of transformation matrices (internal and external) on the orientations of crystals and then we have associated the geometric object 'daughter crystal' and the algebraic object 'coset' into a unique object 'orientational variant'. We have defined the operators as classes of transformations between variants and have identified them as double cosets. We have shown that the set of variants and the set of operators constitute a groupoid, which we have called a groupoid of orientational variants. But what could be the applications and implications of such concepts?

\subsection{Reconstruction of parent grains from EBSD maps}

This theoretical study takes its origin from an applied materials science problem. We initially wanted to determine, from EBSD maps acquired on different phase transition materials ( $\alpha$ titanium alloys used for space applications and $\alpha$ steels used for nuclear applications) the size and the orientation of the parent grains that have produced these variants, i.e. the $\beta$-Ti and $\gamma$-Fe grains, respectively. Some studies of this problem already exist for Burgers transitions; the method consists in calculating the disorientation between two $\alpha$ grains and checking if it corresponds to a 'special misorientation' (i.e. an operator of $\left\langle\mathbf{O}^{\alpha}\right\rangle$ ). As we will explain and quantify in Cayron et al. (2006), the probability of finding an operator between two randomly oriented $\alpha$ crystals is low for Burgers transitions but is far from being negligible for martensitic transitions in steels. The only way to avoid abusive grain regrouping is to check the coherency of all the $\alpha$ crystals considered as variants at each step of the algorithm. This can be performed with the help of the composition table of the groupoid (\$7.3), without solving any equation as in Gey \& Humbert (2003). For example, to know if three adjacent grains are variants inherited from the same parent crystals, one must check (i) if the misorientations between them correspond to ones of the theoretical operators and (ii) if the composition of the operators is coherent. In this way, if the grains $G_{1}, G_{2}$ and $\mathrm{G}_{3}$ are found to be linked by the operators $\mathrm{G}_{12}=\mathrm{O}_{1}^{\alpha}, \mathrm{G}_{13}=\mathrm{O}_{4}^{\alpha}$ and $\mathrm{G}_{23}=\mathrm{O}_{6}^{\alpha}$, then since $\mathrm{G}_{23}=\left(\mathrm{G}_{12}\right)^{-1} \mathrm{G}_{13} \in\left(\mathrm{O}_{1}^{\alpha}\right)^{-1} \mathrm{O}_{4}^{\alpha}$ (see Fig. 6), the triplet of operators is coherent. If grains $G_{1}, G_{2}$ and $\mathrm{G}_{3}$ were found to be linked by the operators $\mathrm{G}_{12}=\mathrm{O}_{1}^{\alpha}, \mathrm{G}_{13}=$ $\mathrm{O}_{4}^{\alpha}$ and $\mathrm{G}_{23}=\mathrm{O}_{1}^{\alpha}$, the triplet is not coherent (see Fig. 6), so we would be able to deduce that these grains are in fact accidentally linked by misorientations close to operators. More details will be given in Cayron et al. (2006).

\subsection{Groupoids and complexity}

It may be noticed that the algebraic structure describing the 'general' case of Fig. 1( $a$ ) is a group and the one describing the 'particular' cases of Figs. 1(b) and (c) is a groupoid, an algebraic structure that is more general than groups. This is a surprising point; indeed, one could expect the opposite and think that 'particular' cases are described by one subfamily of the 'general' case. Two elements of response can be given.

1. If we link an orthogonal basis to the parent cubic crystal and to the triangular daughter crystals (with the help of the structure tensors, for example), $\mathrm{T}_{>}$can be substituted by a rotation matrix $R_{>}$. Then, the number of variants $\mathrm{N}^{\alpha}$ appears as a function of $\phi$, the rotation angle of $R_{>}$. In the case of Fig. 1 , this function is always equal to 8 , except for the rotation angles $\delta=N \pi / 4$ (with $N$ integer), where $\mathrm{N}^{\alpha}$ is then reduced to 4. The function $\mathrm{N}^{\alpha}(\phi)$ appears as a Dirac comb with a period $\mathrm{T}=\pi / 4$. Each Dirac $\delta$ of this comb represents an 'algebraic structure breaking' (with reference to the usual term 'symmetry breaking' used for phase transitions). Therefore, the words 'particular' and 'general' probably do not have any mathematical meaning in this case: Figs. $1(a)$ and $(b)$ are actually two distinct cases.

2. The fact that Fig. 1(a) seems geometrically 'more complex' than Fig. 1(b) comes from its higher number of triangles. However, this is only an apparent complexity. Indeed, the number of components in a system is only one part of its complexity; the interconnections between the components are also very important (see for example Allegrini et al., 2004). One may actually conceive that the structures of Figs. $1(b)$ and $(c)$ are more complex than that of Fig. 1(a) because these structures are 'constrained'; any modification of $\phi$ degenerates the algebraic structure of Figs. 1(b) or $(c)$ (a groupoid) into a simpler one (a group). More studies would be required to clearly define an 'algebraic complexity'.

\subsection{Transition cycles: irreversibility and entropy}

For Landau transitions, as presented in $\$ 8.1$, the number of variants of the $n$th generation is always equal to $1: \mathrm{N}_{2}^{\beta}=\mathrm{N}_{3}^{\beta}=$ 
$\ldots=\mathrm{N}_{\infty}^{\beta}=\mathrm{N}^{\beta}=1$. Consequently, if only the orientations are considered, the system remains algebraically stable (the algebraic structure of the initial crystal, $\mathbf{G}^{\beta}$, and that of orientational variants, $\mathbf{V}^{\alpha}$, are isomorphic), and the physical structure is recovered after one cycle. We conclude therefore that Landau transitions are reversible during thermal cycling. This implies that the irreversible part of the entropy variation at each cycle is zero: $\Delta S_{i}$ (one cycle) $=0$. We recall that the present system is open (and not isolated) and that $\Delta \mathrm{S}=$ $\Delta \mathrm{S}_{e}+\Delta \mathrm{S}_{i}$ where $\Delta \mathrm{S}_{e}$ is the flow of entropy due to the heat exchanges and $\Delta S_{i}$ is the entropy production owing to irreversible processes inside the system (Prigogine et al., 1972).

However, the situation is completely different for reconstructive transitions. For example, for Burgers transitions, one monocrystal $\beta_{1}$ can be transformed by cooling into 12 variants by $\beta_{1} \rightarrow \alpha_{i}$ and, if we re-heat the material, each variant becomes the parent crystal for the transition $\alpha_{i} \rightarrow \beta_{k,}$ such that the number of variants of second generation is $\mathrm{N}_{2}^{\beta}=40$ (see §8.3). At each cycle of reconstructive transitions, more and more variants are introduced, the complexity of the system increases and the structure will never recover. Consequently, we find algebraically that the reconstructive transitions are intrinsically irreversible. The irreversible part of the entropy variation on the first cycle is strictly positive: $\Delta S_{i}(1$ st cycle $)>0$. For the moment, no general formula that gives $\mathrm{N}_{2}^{\beta}$ could have been proposed. But is it possible to find such a formula? More generally, how many variants do we obtain after $N$ cycles? What is their algebraic structure? The number of distinct crystals increases after each cycle $\left(\mathrm{N}^{\beta} \leq \ldots \leq \mathrm{N}_{n}^{\beta} \leq \mathrm{N}_{n+1}^{\beta}\right)$, but does it stabilizes for an $n$th cycle? This would imply that $\Delta \mathrm{S}_{i}(n$th cycle $)=0$. This problem constitutes a fascinating algebraic subject. Experimentally speaking, if we heat and cool a phase-transition material around its transition temperatures (in order to decrease its grain size and increase its mechanical properties) and perform X-ray diffraction, will we obtain an isotropic (random) or textured pattern after a very high number of cycles? Geometrically speaking, if we assume that the crystals keep their size, we must study how to make a three-dimensional pavement with two kinds of solids like in a Penrose problem. If we assume that the variants are smaller and smaller at each cycle (such as presented in Fig. 4), the different generated variants will form a threedimensional fractal. For example, we will show in another study (Cayron, 2006) that the $\Sigma 3^{n}$ multiple twinning in cubic materials can be algebraically represented by a groupoid and geometrically by a three-dimensional fractal.

We recall that the complexity of the structure produced by reconstructive transitions comes from the fact that the subgroup $\mathbf{H}^{\beta}$ is not in general a normal group in $\mathbf{G}^{\beta}$, which is a consequence of the non-commutativity between the transformation matrices. Therefore, it could be interesting to see if some mathematical concepts developed in non-commutative geometry (Connes, 1990) could be applied to describe mathematically the transition cycles.

\section{Conclusions}

In this study, $\alpha$ daughter crystals in an orientation relationship with a $\beta$ parent crystal have been defined from their orientations in a fixed reference basis with the help of transformation matrices. They can be created by $\beta \rightarrow \alpha$ phase transitions (Landau or reconstructive), by twinning or by precipitation. We called $\mathrm{T}_{>}$one of the possible transformation matrices from a basis of a parent crystal $\beta$ to a basis of a daughter crystal $\alpha_{1}$. Two equivalence classes on the transformation matrices have been introduced: an internal class for the matrices that point $t o$ the same crystal $\alpha_{i}$ and an external class for the matrices that point from the same crystal $\beta$. The set of internally and externally equivalent transformation matrices are $T_{>} \mathbf{G}^{\alpha}$ and $\mathbf{G}^{\beta} \mathrm{T}_{>}$, respectively. These two classes are equal for matrices of the intersection group $\mathbf{H}^{\beta}=\mathbf{G}^{\beta} \cap \mathrm{T}_{>} \mathbf{G}^{\alpha} \mathrm{T}_{>}^{-1}$. The set of distinct daughter crystals $\alpha_{i}$, denoted $\mathbf{V}^{\alpha}$, has been identified as the quotient set $\mathbf{G}^{\beta} / \mathbf{H}^{\beta}$. In this way, we have associated the geometric notion of orientational variant $\alpha_{i}$ with the algebraic notion of left coset $\mathrm{g}_{i}^{\beta} \mathbf{H}^{\beta}$ and created a unique concept: the orientational variants. Their number directly results from the Lagrange formula $\mathrm{N}^{\alpha}=\left|\mathbf{G}^{\beta}\right| /\left|\mathbf{H}^{\beta}\right|$. By considering the $\beta \rightarrow \alpha$ and $\alpha \rightarrow \beta$ transitions, we proved the isomorphism $\left|\mathbf{H}^{\alpha}\right| \cong\left|\mathbf{H}^{\beta}\right|$, and hence, that $\mathrm{N}^{\alpha}\left|\mathbf{G}^{\alpha}\right|=\mathrm{N}^{\beta}\left|\mathbf{G}^{\beta}\right|$. The transformations from one variant to another were partitioned into distinct equivalence classes. The set formed by these classes is isomorphic to the set of the left $\mathbf{H}^{\beta}$ orbits on the quotient set $\mathbf{G}^{\beta} / \mathbf{H}^{\beta}$, which is the double quotient set $\mathbf{H}^{\beta} \backslash \mathbf{G}^{\beta} / \mathbf{H}^{\beta}$. These orbits were called 'operators'. Their number is given by the Burnside formula. The set of orientational variants associated with the set of operators constitutes a groupoid. We have proposed a composition table for this groupoid that acts as a crystallographic signature of the phase transition. A method to determine if two daughter variants $\alpha_{i}$ and $\alpha_{j}$ can be inherited from more than one parent crystal was given by calculating the intersection of external orbits. A computer program has been written to determine the variants, the operators, the composition table of the groupoid and the possible parent crystals for any structural transition. Some results obtained with the Burgers transition and with the martensitic transition in steels are given and compared to published results. This work opens the way for deeper algebraic researches on fractal structures formed by reconstructive phase-transition materials after thermal cycling. The complexity, irreversibility and entropy of such systems were briefly discussed.

\section{APPENDIX $A$ \\ Elements of group theory}

An equivalence relation $\sim$ is a relation among elements $a$ of a set $\mathbf{X}$ satisfying: $(a)$ reflexivity: $a \sim a$; $(b)$ symmetricity: $a \sim b$ $\Rightarrow b \sim a$; $(c)$ transitivity: $a \sim b, b \sim c \Rightarrow a \sim c$. An important property of an equivalence relation is that it partitions the set $\mathbf{X}$ into distinct equivalence classes $\mathbf{C}_{a}=\{b \in \mathbf{X}, b \sim a\}$ such that for any $(a, b) \in \mathbf{X}^{2}$ either $\mathbf{C}_{a}=\mathbf{C}_{b}$ or $\mathbf{C}_{a} \cap \mathbf{C}_{b}=\emptyset$. It may be noted that this property is not linked to the algebraic structure of the set $\mathbf{X}$. 
A group is a nonempty set $\mathbf{G}$ together with a law of composition $(a, b) \rightarrow(a b): \mathbf{G} \times \mathbf{G} \rightarrow \mathbf{G}$ satisfying the following axioms: (i) associativity: for all $a, b, c \in \mathbf{G},(a b) c=$ $a(b c)$; (ii) existence of an identity element: $\exists e \in \mathbf{G}$ such that for all $a \in \mathbf{G}$ : $a e=e a=a$; and (iii) existence of inverses: for all $a \in \mathbf{G}, \exists a^{-1} \in \mathbf{G}$ such that $a a^{-1}=a^{-1} a=e$. We will use $|\mathbf{G}|$ to denote, for a finite group $\mathbf{G}$, the number of elements of $\mathbf{G}$, also called order of $\mathbf{G}$. A subgroup $\mathbf{H}$ on a group $\mathbf{G}$, denoted $\mathbf{H} \leq$ $\mathbf{G}$, is a nonempty subset of $\mathbf{G}$ such that $(a)$ for $(a, b) \in \mathbf{H}^{2}, a b \bar{\epsilon}$ $\mathbf{H}$ and $(b)$ for $a \in \mathbf{H}, a^{-1} \in \mathbf{H}$. When specifying a groupsubgroup relation, the sign $\leq$ is preferred to $\subset$ because a set $\mathbf{X}$ can be included in a group $\mathbf{G}$ without being itself a subgroup of $\mathbf{G}$.

A homomorphism from a group $(\mathbf{G},$.$) to a second group$ $\left(\mathbf{G}^{\prime},{ }^{*}\right)$ is a map $\alpha: \mathbf{G} \rightarrow \mathbf{G}^{\prime}$ such that, for all $a, b \in \mathbf{G}, \alpha(a b)=$ $\alpha(a)^{*} \alpha(b)$. The image of $\alpha$ is defined as $\operatorname{Im}(\alpha)=\left\{g^{\prime} \in \mathbf{G}^{\prime}, \exists g \in\right.$ $\mathbf{G}$ with $\left.g^{\prime}=\alpha(g)\right\}$. It is a subgroup of $\mathbf{G}^{\prime}$. The kernel of $\alpha$ is defined as $\operatorname{Ker}(\alpha)=\left\{g \in \mathbf{G}, \alpha(g)=e^{\prime}\right\}$ with $e^{\prime}$ being the identity element of $\mathbf{G}^{\prime}$. It is a subgroup of $\mathbf{G}$. An isomorphism between $\mathbf{G}$ and $\mathbf{G}^{\prime}$ is simply a bijective homomorphism (a one-to-one map between $\mathbf{G}$ and $\left.\mathbf{G}^{\prime}\right) . \alpha$ is an isomorphism if and only if $\operatorname{Ker}(\alpha)=\{e\}$ and $\operatorname{Im}(\alpha)=\mathbf{G}^{\prime}$.

Let $\mathbf{H}$ be a subgroup of $\mathbf{G}$. For any fixed $a \in \mathbf{G}$, a left coset of $\mathbf{H}$ in $\mathbf{G}$ is defined by the set of the form $a \mathbf{H}=\{a h$ for $h \in \mathbf{H}\}$. Similarly, a right coset is defined by $\mathbf{H} a=\{h a$ for $h \in \mathbf{H}\}$. It can be checked that (i) two left cosets are either disjoint or equal, that (ii) $a \mathbf{H}=b \mathbf{H}$ if and only if $a^{-1} b \in \mathbf{H}$, and that (iii) any two left cosets have the same number of elements equal to $|\mathbf{H}|$. Similarly, two right cosets $\mathbf{H} a=\mathbf{H} b$ if and only if $a b^{-1} \in \mathbf{H}$.

The left and right cosets of $\mathbf{H}$ in $\mathbf{G}$ partition $\mathbf{G}$. The condition ' $a$ and $b$ lie in the same left coset' is a left class of equivalence. We denote $\mathbf{G} / \mathbf{H}$ the set of all the left cosets and $\mathbf{G} \backslash \mathbf{H}$ the set of all the right cosets. $\operatorname{Card}(\mathbf{G} / \mathbf{H})$, denoted $|\mathbf{G} / \mathbf{H}|$ or $[\mathbf{G}: \mathbf{H}]$, is the number of left cosets of $\mathbf{H}$ in $\mathbf{G}$, it is also equal to the number of right cosets. A direct consequence of the partitioning of $\mathbf{G}$ into left equivalence classes is the Lagrange formula: for any $\mathbf{H}$ subgroup of a finite group $\mathbf{G}$, the order of $\mathbf{H}$ divides the order of $\mathbf{G}$, i.e. $|\mathbf{G}|=|\mathbf{G} / \mathbf{H}||\mathbf{H}|$.

Two subgroups $\mathbf{H}_{1}$ and $\mathbf{H}_{2}$ are conjugate if $\exists g \in \mathbf{G}$ such that $\mathbf{H}_{1}=g \mathbf{H}_{2} g^{-1}$. One may verify that the property 'being conjugate' constitutes an equivalent class. A subgroup $\mathbf{N}$ of $\mathbf{G}$ is normal (or invariant, or self-conjugated), denoted $\mathbf{N} \triangleleft \mathbf{G}$, if and only if, for all $g \in \mathbf{G}, g \mathbf{N} g^{-1}=\mathbf{N}$. This condition is also equivalent to: for all $g \in \mathbf{G}, g \mathbf{N}=\mathbf{N} g$ or to: for all $g \mathbf{N}$ in $\mathbf{G} / \mathbf{N}$, $(g \mathbf{N})^{-1}=\mathbf{N} g^{-1}$. In other words, for a normal subgroup $\mathbf{N}$, each left coset of $\mathbf{N}$ in $\mathbf{G}$ is equal to a right coset of $\mathbf{N}$ in $\mathbf{G}$. The kernel of a homomorphism $\alpha: \mathbf{G} \rightarrow \mathbf{G}^{\prime}$ is a normal subgroup of G. If $\mathbf{N} \triangleleft \mathbf{G}$, a natural composition law '.' can be defined on $\mathbf{G}$ / $\mathbf{N}$ the set of cosets of $\mathbf{N}$ in $\mathbf{G}$ by

$$
\text { for } a \mathbf{N} \text { and } b \mathbf{N} \in \mathbf{G} / \mathbf{N}:(a \mathbf{N})(b \mathbf{N})=(a b) \mathbf{N} \text {. }
$$

Indeed, the law '.' is well defined (i.e. it does not depend on the choice of the representative $a$ in the equivalence class $a \mathbf{N}$ ) and it confers on $\mathbf{G} / \mathbf{N}$ a natural group structure. Reciprocally, a natural group structure exists on $\mathbf{G} / \mathbf{N}$ only if $\mathbf{N} \triangleleft \mathbf{G}$. In this case, $\mathbf{G} / \mathbf{N}$ is called the quotient group.
There are many important isomorphism theorems in group theory that will not be described here. One of their corollaries is the decomposition of a group into a direct product of two subgroups - see for example Milne (2003): Consider two subgroups $\mathbf{H}$ and $\mathbf{K}$ of a group $\mathbf{G}$ such that $\mathbf{G}=\mathbf{H K}=\{h k, h \in$ $\mathbf{H}, k \in \mathbf{K}\}$ and $\mathbf{H} \cap \mathbf{K}=\{e\}$. Then:

(a) if $\mathbf{H} \triangleleft \mathbf{G}$, then $\mathbf{G} / \mathbf{H}$ is isomorphic to $\mathbf{K}$; there is a one-toone correspondence between the left cosets and the elements of $\mathbf{K}$;

(b) if $\mathbf{H} \triangleleft \mathbf{G}$ and if $\mathbf{K} \triangleleft \mathbf{G}$, then $\mathbf{G}$ is isomorphic to $\mathbf{H} \times \mathbf{K}=$ $\{(h, k), h \in \mathbf{H}, k \in \mathbf{K}\}$; the group $\mathbf{G}$ can be canonically decomposed as the product of the two subgroups $\mathbf{H}$ and $\mathbf{K}$.

\section{APPENDIX $B$ \\ Groups and actions on sets}

Let us now introduce some notions on groups acting on sets. Let $\mathbf{X}$ be a set and $\mathbf{G}$ be a group. A left action of $\mathbf{G}$ on $\mathbf{X}$, denoted '.', is a map $(g, x) \rightarrow$ g. $x:(\mathbf{G}, \mathbf{X}) \rightarrow \mathbf{X}$ such that: $e . x=x$ for all $x \in \mathbf{X}$ and (gh). $x=g(h . x)$ for all $g h \in \mathbf{G}$ and $x \in \mathbf{X}$.

For $x$ and $y \in \mathbf{X}$, we write $x \sim_{\mathbf{G}} y$ if and only if $\exists g \in \mathbf{G}$ such that $y=$ g.x. It can be checked that this relation is an equivalence relation on $\mathbf{X}$. The left equivalence classes are called the left $\mathbf{G}$ orbits. For a fixed $x \in \mathbf{X}$, we denote the left $\mathbf{G}$ orbit containing $x$ as

$$
\mathrm{O}_{x}=\{g \cdot x \text { for } g \in \mathbf{G}\} \text {. }
$$

Note that all the distinct $\mathbf{G}$ orbits partition $\mathbf{X}$. The group $\mathbf{G}$ is said to act transitively on $\mathbf{X}$ if there is only one orbit, i.e. if, $\forall x, y \in \mathbf{X}, \exists g \in \mathbf{G} / y=g . x$.

Let us call for any $x \in \mathbf{X}$, the stabilizer at the left of $x$ :

$$
\operatorname{Stab}_{\mathbf{G}}(x)=\{g \in \mathbf{G}, g \cdot x=x\} .
$$

It is a subgroup of $\mathbf{G}$, also called the isotropy group. One can then prove that, if $\mathbf{G}$ acts at the left on $\mathbf{X}$, then the application defined by $g \in \mathbf{G}$ and $x \in \mathbf{X}$,

$$
\begin{aligned}
\mathbf{G} / \operatorname{Stab}_{\mathbf{G}}(x) & \rightarrow \mathrm{O}_{x} \\
g \operatorname{Stab}_{\mathbf{G}}(x)=\left\{g g^{\prime}, g^{\prime} \in \mathbf{S t a b}_{\mathbf{G}}(x)\right\} & \rightarrow g \cdot x,
\end{aligned}
$$

is a bijection. Therefore, $\left|\mathrm{O}_{x}\right|=|\mathbf{G}| /\left|\mathbf{S t a b}_{\mathbf{G}}(x)\right|$.

We call $\mathrm{O}_{i}$ all the $\mathbf{G}$ orbits in $\mathbf{X}$ and $N_{o}$ their number: $\mathbf{X}=\bigcup_{i=1}^{N_{o}} \mathrm{O}_{i}$.

If we choose for each $\mathrm{O}_{i}$ a point $x_{i} \in \mathbf{X}$ such that $\mathrm{O}_{i}=\mathrm{O}_{x_{i}}$, we obtain the class equation:

$$
\begin{aligned}
|\mathbf{X}| & =\sum_{i=1}^{N_{o}}\left[\mathbf{G}: \operatorname{Stab}_{\mathbf{G}}\left(x_{i}\right)\right] \\
& =\sum_{i=1}^{N_{o}} n_{i} \quad \text { with } n_{i}=|\mathbf{G}| /\left|\operatorname{Stab}_{\mathbf{G}}\left(x_{i}\right)\right| \in \mathbb{N} .
\end{aligned}
$$

Now let us introduce, for $g \in \mathbf{G}, \mathbf{F i x}_{\mathbf{X}}(g)=\{x \in \mathbf{X} / g \cdot x=x\}$. Then 


$$
\begin{aligned}
\sum_{g \in \mathbf{G}}\left|\mathbf{F i x}_{\mathbf{X}}(g)\right| & =\sum|\{(g, x) \in(\mathbf{G} \times \mathbf{X}), g . x=x\}| \\
& =\sum_{x \in \mathbf{X}}\left|\mathbf{S t a b}_{\mathbf{G}}(x)\right|=\sum_{x \in \mathbf{X}} \frac{|\mathbf{G}|}{\left|\mathrm{O}_{x}\right|}=\sum_{O_{i} \subset \mathbf{X}}|\mathbf{G}| \sum_{x_{i} \in \mathrm{O}_{i}} \frac{1}{\left|\mathrm{O}_{x_{i}}\right|} \\
& =|\mathbf{G}| \sum_{\mathrm{O}_{i} \subset \mathbf{X}} 1 .
\end{aligned}
$$

We obtain the Burnside formula giving the number of distinct orbits by

$$
N_{o}=\frac{1}{|\mathbf{G}|} \sum_{g \in \mathbf{G}}\left|\mathbf{F i x}_{\mathbf{X}}(g)\right| .
$$

The number of distinct orbits $N_{o}$ is the average of the elements fixed by the orbits.

For a subgroup $\mathbf{H}$, the set of all $g \in \mathbf{G}$ such that $g \mathbf{H} g^{-1}=\mathbf{H}$ is called the normalizer of $\mathbf{H}$ in $\mathbf{G}$ and is denoted $\mathbf{N}_{\mathbf{G}}(\mathbf{H})$. For a normal subgroup of $\mathbf{G}, \mathbf{N}_{\mathbf{G}}(\mathbf{N})=\mathbf{G}$. Clearly, $\mathbf{N}_{\mathbf{G}}(\mathbf{H})$ is the largest subgroup of $\mathbf{G}$ containing $\mathbf{H}$ as a normal subgroup. We also call $\mathbf{N}_{0}(\mathbf{H})=\bigcap_{g \in \mathbf{G}} g \mathbf{H} g^{-1}$. It has been proved, see for example Milne (2003), that $\mathbf{N}_{0}(\mathbf{H})$ is the largest normal subgroup contained in $\mathbf{H}$.

Let us now consider two interesting examples of the class equation (which will be used in the present study).

1. With $\mathbf{X}=\{\mathbf{H}, \mathbf{H} \leq \mathbf{G}\}$ the set of all the subgroups of $\mathbf{G}$, then $\mathbf{G}$ acts on $\mathbf{X}$ by conjugation: for any $g \in \mathbf{G}, g . \mathbf{H}=g \mathbf{H} g^{-1}$. One may check that for this action the stabilizer is $\operatorname{Stab}_{\mathbf{G}}^{\mathrm{c}}(\mathbf{H})=$ $\mathbf{N}_{\mathbf{G}}(\mathbf{H})$. The superscript c means 'by conjugation'. For a fixed subgroup $\mathbf{H}$ of $\mathbf{G}$, the number of distinct subgroups that are conjugate is given by the class equation applied to $\mathbf{X}=\left\{g \mathbf{H}^{-1}\right.$, $g \in \mathbf{G}\}$, it is $|\mathbf{G}| /\left|\mathbf{N}_{\mathbf{G}}(\mathbf{H})\right|$.

2. With $\mathbf{X}=\mathbf{G} / \mathbf{H}$, the partition formed by the left $\mathbf{H}$ cosets on $\mathbf{G}$, then:

(a) $\mathbf{G}$ acts on $\mathbf{G} / \mathbf{H}$ by the classical left product: for any $g^{\prime} \in$ $\mathbf{G}, g^{\prime} \cdot g \mathbf{H}=g^{\prime} g \mathbf{H}$. Note that, for this action, the stabilizer is $\mathbf{S t a b}_{\mathbf{G}}(g \mathbf{H})=g \mathbf{H} g^{-1}$, the conjugate of $\mathbf{H}$ by $g$ and that in general $\mathbf{S t a b}_{\mathbf{G}}(\mathbf{H}) \neq \mathbf{S t a b}_{\mathbf{G}}^{\mathrm{c}}(\mathbf{H})$ unless $\mathbf{H} \triangleleft \mathbf{G}$. The class equation applied to $\mathbf{G} / \mathbf{H}$ is equivalent to Lagrange's formula.

(b) $\mathbf{H}$ acts at the left of $\mathbf{G} / \mathbf{H}$ : for any $h \in \mathbf{H}, h \cdot g \mathbf{H}=h g \mathbf{H}$. Note that, for this operation, the stabilizer is $\operatorname{Stab}_{\mathbf{H}}(g \mathbf{H})=$ $\mathbf{H} \cap g \mathbf{H} g^{-1}$.

Thus, for any $\mathbf{H} \leq \mathbf{G}$ and $g \in \mathbf{G}$,

$$
\{e\} \leq \mathbf{N}_{0}(\mathbf{H}) \leq \operatorname{Stab}_{\mathbf{H}}(g \mathbf{H}) \leq \mathbf{H} \leq \mathbf{N}_{\mathbf{G}}(\mathbf{H}) \leq \mathbf{G}
$$

and, if $\mathbf{H}=\mathbf{N} \triangleleft \mathbf{G}$,

$$
\mathbf{N}_{0}(\mathbf{H})=\mathbf{S t a b}_{\mathbf{H}}(g \mathbf{H})=\mathbf{H} \text { and } \mathbf{N}_{G}(\mathbf{H})=\mathbf{G} .
$$

We are very grateful to Professor Michéa for having pointed our attention to the special mathematical concept of groupoid. We also thank him for checking some algebraic developments of the paper. We are grateful to Dr Jouneau for his precious advice on crystallography and his help in the Python language. Acknowledgments are also due to Dr Briottet for supporting this work. The notations have been simplified and the bibliography has been improved thanks to the referees' remarks.

\section{References}

Akbay, B., Aydinol, K. \& Bor, A. S. (1994). J. Appl. Cryst. 27, 379-384.

Allegrini, P., Giuntoli, M., Grigolini, P. \& West, B. J. (2004). Chaos, Solitons Fractals, 20, 11-32.

Bollmann, W. (1970). Crystal Defects and Crystalline Interfaces. Berlin: Springer-Verlag.

Bollmann, W. (1982). Crystal Lattices, Interfaces, Matrices. Published by the author.

Bradley, C. J. \& Cracknell, A. P. (1972). The Mathematical Theory of Symmetry in Solids. Oxford: Clarendon Press.

Brandt, W. (1926). Math. Ann. 96, 360-366.

Burgers, W.G. (1934). Physica (Utrecht), 1, 561-569.

Cahn, J. W. \& Kalonji, G. (1981). Solid-Solid Phase Transformations, edited by H. I. Aaronson, D. E. Laughlin, R. F. Skerka \& C. M. Marvin Wayman, pp. 3-14. Washington: The Metallurgical Society of AIME.

Cayron, C. (2000). PhD thesis No. 2246, EPFL, Lausanne, Switzerland, http://cimewww.epfl.ch/people/cayron/these.html.

Cayron, C. (2006). In preparation for Acta Cryst. A.

Cayron, C., Artaud, B. \& Briottet, L. (2006). Submitted to Mater. Charact.

Connes, A. (1990). Géométrie Non Commutative. Paris: Dunod (Inter-Editions U).

Curie, P. (1894). J. Phys. 3, 393-415.

Dirl, R., Zeiner, P., Davies, B. L. \& Janovec, V. (1997). Acta Cryst. A53, 456-466.

Dornberger-Schiff, K. (1959). Acta Cryst. 12, 246.

Dornberger-Schiff, K. \& Grell-Niemann, H. (1961). Acta Cryst. 14, 167-177.

Friedel, G. (1904). Étude sur les Groupements Cristallins. Extrait du Bulletin de la Société de l'Industrie Minérale, Quatrième Série, Tomes III et IV. Saint-Étienne: Société de l'Imprimerie Thèolier J. Thomas et C.

Gey, N. \& Humbert, M. (2003). J. Mater. Sci. 38, 1289-1294.

Gottstein, G. (1984). Acta Metall. 32, 1117-1138.

Gourgues, A. F., Flower, H. M. \& Lindley, T. C. (2000). Mater. Sci. Tech. 16, 26-40.

Gratias, D. \& Portier, R. (1982). J. Phys. 43, C6, 15-24.

Grimmer, H., Bollmann, W. \& Warrington, D. H. (1974). Acta Cryst. A30, 197-207.

Guymont, M. (1981). Phys. Rev. B, 24, 2647-2655.

Hahn, Th. \& Klapper, H. (2003). International Tables for Crystallography, Vol. D, edited by A. Authier, Section 3.3, pp. 393-448. Dordrecht: Kluwer Academic Publishers.

Humbert, M. \& Gey, N. (2002). J. Appl. Cryst. 35, 401-405.

Humbert, M., Wagner, F. \& Esling, C. (1992). J. Appl. Cryst. 25, 724-730.

Humbert, M., Wagner, F., Moustahfid, H. \& Esling, C. (1995). J. Appl. Cryst. 28, 571-576.

Janovec, V. (1972). Csech. J. Phys. B22, 974-994.

Janovec, V. (1976). Ferroelectrics, 12, 43-53.

Janovec, V. \& Dvorakova, E. (1989). Acta Cryst. A45, 801-802.

Janovec, V., Hahn, Th. \& Klapper, H. (2003). International Tables for Crystallography, Vol. D, edited by A. Authier, Section 3.2, pp. 377-391. Dordrecht: Kluwer Academic Publishers.

Janovec, V. \& Přívratská, J. (2003). International Tables for Crystallography, Vol. D, edited by A. Authier, Section 3.4, pp. 449-505. Dordrecht: Kluwer Academic Publishers.

Kalonji, G. (1985). J. Phys. 46, C4, 249-256.

Kargapolov, M. \& Merzliakov, I. (1985). Eléments de la Théorie des Groupes. Moscow: Mir.

Kitahara, H., Ueji, R., Ueda, M., Tsuji, N. \& Minamino, Y. (2005). Mater. Charact. 54, 378-386.

Kocks, U. F., Tomé, C. N. \& Wenk, H. R. (1998). Texture and Anisotropy. edited by U. F. Kocks, pp. 45-102. Cambridge University Press. 


\section{research papers}

Kurdjumov, G. \& Sachs, G. (1930). Z. Phys. 64, 325-343.

Landau, L. D. (1937a). Phys. Z. Sowjun. 11, 26-47.

Landau, L. D. (1937b). Phys. Z. Sowjun. 11, 545-555.

Marteli, A. (2004). Python en Concentré. Paris: O'Reilly.

Milne, J. S. (2003). Group Theory, http://www.jmilne.org/math/.

Morito, S., Tanaka, H., Konishi, R., Furuhara, T. \& Maki, T. (2003). Acta Mater. 51, 1789-1799.

Nishiyama, Z. (1934). Sci. Rep. Tohoku Imp. Univ. 23, 637-644.

Prigogine, I., Nicolis, G. \& Babloyantz, A. (1972). Phys. Today, 25, 23-28.

Sadanaga, R. (1978). Rec. Prog. Nat. Sci. Jpn, 3, 143-151.

Sadanaga, R. \& Ohsumi, K. (1979). Acta Cryst. A35, 115-122.
Standford, N. \& Bate, P. S. (2005). Acta Mater. 53, 859-867.

Stewart, I. (2004). Nature (London), 427, 601-604.

Tolédano, J. C. \& Tolédano, P. (1987). The Landau Theory of Phase Transition. Singapore: World Scientific.

Tolédano, P. \& Dmitriev, V. (1996). Reconstructive Phase Transitions. Singapore: World Scientific.

Van Tendeloo, G. \& Amelinckx, S. (1974). Acta Cryst. A30, 431-440.

Wadhawan, V. K. (1997). Acta Cryst. A53, 546-555.

Warrington, D. H. \& Bufalini, P. (1971). Scr. Metall. 5, 771-776.

Wassermann, G. (1933). Arch Eisenhüttenwes. 16, 149-155.

Weinstein, A. (1996). Am. Math. Soc. 43, 744-752.

Yamamoto, A. \& Ishihara, K. N. (1988). Acta Cryst. A44, 707-714. 